\title{
Title
}

\section{Expert-integrated automated machine learning uncovers hemodynamic predictors in spinal cord injury}

\author{
Austin Chou ${ }^{1,2,3}$, Abel Torres-Espin ${ }^{1,2,3}$, Nikos Kyritsis ${ }^{1,2,3}$, J. Russell Huie ${ }^{1,2,3}$, Sarah Khatry ${ }^{4}$, Jeremy Funk ${ }^{4}$, \\ Jennifer Hay ${ }^{4}$, Andrew Lofgreen ${ }^{4}$, Rajiv Shah ${ }^{4}$, Chandler McCann ${ }^{4}$, Lisa U Pascual ${ }^{5}$, Edilberto Amorim ${ }^{3,6}$, \\ Philip R Weinstein ${ }^{2,6,7}$, Geoffrey T Manley ${ }^{1,2,3}$, Sanjay S Dhall ${ }^{1,2,3}$, Jonathan Z Pan ${ }^{1,8}$, Jacqueline C \\ Bresnahan $^{1,2,3}$, Michael S Beattie ${ }^{1,2,3}$, William D Whetstone ${ }^{9}$, Adam R Ferguson*, ${ }^{* 1,2,3}$, and the TRACK-SCI \\ Investigators $^{10}$ \\ ${ }^{1}$ Weill Institute for Neurosciences, Brain and Spinal Injury Center, University of California, San Francisco \\ (UCSF) \\ ${ }^{2}$ Department of Neurological Surgery, University of California, San Francisco (UCSF) \\ ${ }^{3}$ Zuckerberg San Francisco General Hospital and Trauma Center \\ ${ }^{4}$ DataRobot, Inc., Boston, MA, USA. \\ ${ }^{5}$ Orthopaedic Trauma Institute, Department of Orthopaedic Surgery, University of California, San \\ Francisco (UCSF) \\ ${ }^{6}$ Department of Neurology, University of California, San Francisco (UCSF) \\ ${ }^{7}$ Weill Institute for Neurosciences, Institute for Neurodegenerative Diseases, Spine Center, University of \\ California, San Francisco (UCSF) \\ ${ }^{8}$ Department of Anesthesia and Perioperative Care, University of California, San Francisco (UCSF) \\ ${ }^{9}$ Department of Emergency Medicine, University of California, San Francisco (UCSF) \\ ${ }^{10}$ Consortia authorship in alphabetic order: TRACK-SCI Investigators \\ Beattie MS, Bresnahan JC, Burke JF, Chou A, de Almeida CA, Dhall SS, DiGiorgio AM, Doung-Fernandez X, \\ Ferguson AR, Haefeli J, Hemmerle DD, Huie JR, Kyritsis N, Manley GT, Moncivais S, Omondi C, Pan JZ, \\ Pascual LU, Singh V, Talbott JF, Thomas LH, Torres-Espin A, Weinstein P, Whetstone WD
}

* Corresponding Author:

Adam R Ferguson, Ph.D.

Professor

Zuckerberg San Francisco General Hospital

1001 Potrero Ave, Building 1, Room 101

San Francisco, CA 94110

Tel.: +1-628-206-3708

Email: Adam.Ferguson@ucsf.edu 


\section{Abstract}

Automated machine learning (AutoML) is positioned to democratize artificial intelligence (Al) by reducing the amount of human input and $\mathrm{ML}$ expertise needed to create prediction models. However, successful translation of $\mathrm{ML}$ in biomedicine requires moving beyond optimizing only for prediction accuracy and towards discovering reproducible clinical and biological inferences. Here, we present a model-agnostic framework to reinforce AutoML using strategies and tools of explainable and reproducible $\mathrm{Al}$, including novel metrics for performance precision and feature instability. The framework enables clinicians to interpret AutoML-generated models for clinical and biological verifiability and consequently integrate domain expertise during model development. We applied the framework towards spinal cord injury prognostication and identified a detrimental relationship between intraoperative hypertension and patient outcome. Furthermore, our analysis captured evolving clinical practices such as faster time-to-surgery and blood pressure management that affected clinical model validation. Altogether, we illustrate how augmenting AutoML for inferential reproducibility empowers biomedical discovery and builds trust in Al processes towards effective clinical integration.

\section{Introduction}

Automated machine learning (AutoML) is a rapidly-developing $\mathrm{ML}$ subfield focused on automating model optimization processes including algorithm selection, feature engineering, and hyperparameter tuning $^{1,2}$. AutoML applications produce high-performance models across diverse sophisticated algorithms and preprocessing methodologies while reducing the overall need for human input and modeling expertise $\mathrm{e}^{3,4}$. Correspondingly, AutoML lowers the technical and knowledge barrier impeding $M L$ democratization for various domains including biomedicine $e^{5-9}$. With the growing popularity of $M L$ in clinical research ${ }^{10-13}$ and the increasing breadth, depth, and accessibility of clinical health data ${ }^{14}$, AutoML stands to exponentially accelerate clinical ML applications by empowering scientists and clinicians to train and leverage powerful models ${ }^{5}$. However, clinical utility requires ML models to be interpretable for biological mechanisms, verifiable by clinicians, and methodologically and inferentially reproducible ${ }^{15,16}$. Achieving reproducibility is further complicated by the fact that clinical datasets often have small sample sizes relative to the number of variables collected which can result in unstable model behavior ${ }^{17,18}$. This is especially true for rare diseases with smaller patient populations, and translation of AutoML from computer-to-clinic thus necessitates additional approaches beyond maximizing prediction accuracy with "black box" algorithms. In this study, we applied a modeling framework incorporating explainable and reproducible Al strategies to predict spinal cord injury $(\mathrm{SCl})$ patient outcome. Furthermore, we demonstrate how we can improve the inferential reproducibility of $M L$ and integrate clinical expertise in the process while leveraging AutoML for model optimization.

SCls are highly debilitating, resulting in chronic motor, sensory, and autonomic impairment including paralysis. While $\mathrm{SCl}$ affects comparatively fewer patients - about 17,700 new cases a year and 291,000 patients with chronic disabilities in the US alone - the total societal cost is estimated to exceed \$267 billion ${ }^{19,20}$. The variety of $\mathrm{SCl}$ characteristics makes identification of patient outcome predictors challenging despite the volume of data collected throughout the hospitalization and treatment of each patient ${ }^{20}$. Various prognostic models for $\mathrm{SCl}$ outcome have been developed with algorithms ranging from logistic regression to extreme gradient boosted (XGB) trees and convolutional neural networks ${ }^{12,21,22}$. While such studies bear potential for informing clinical care, algorithm selection in many 
SCI ML studies have primarily depended on the researchers' familiarity with specific ML algorithms, and prediction accuracy remains the primary metric for comparing models ${ }^{23,24}$. Moreover, deciphering the relationships between outcome and predictors is still difficult with complex algorithms, ultimately dampening clinician enthusiasm about applying ML tools and results given the inability to interpret and verify such models ${ }^{25}$.

In our AutoML application for $\mathrm{SCl}$ patient prognosis, we demonstrate:

- A framework for reproducible and explainable modeling that implements (1) a repeated crossvalidation strategy, (2) performance precision and feature instability metrics and analyses, (3) model interpretation with permutation feature importance $(\mathrm{pFI})$ and partial dependence plots (PDPs), (4) stabilized backward feature reduction, and (5) model validation with population similarity analysis (Fig. 1). In particular, repeated cross-validation allows for model aggregation to account for modeling variability and improve the inferential reproducibility of the results.

- The importance of integrating domain expertise. We highlight how stabilized pFI and PDPs, useful model-agnostic explainable Al tools, enable biomedical researchers to draw robust inferences regarding the relationship between clinical variables and outcome. Furthermore, we illustrate how augmenting feature selection with domain expertise can improve model performance beyond deploying ML naively.

- Additional analyses to interpret model validity. Many clinical ML studies with smaller sample sizes depend on newly collected data for external validation. Investigating the population similarity between training and validation cohorts provides meaningful information about model generalizability beyond validation performance and can capture evolving clinical practices that inevitably affects clinical ML implementation.

By applying this framework to $\mathrm{SCl}$, we identified actionable intraoperative mean arterial pressure (MAP) thresholds for hypertension and hypotension associated with worse patient outcome. Additionally, our analysis revealed underlying shifting clinical practices such as reducing time-to-surgery and hypotension management that invariably affects model validation efforts in $\mathrm{SCl}$ research. Altogether, we present methods to bolster the interpretability, reproducibility, and trustworthiness of clinical ML as AutoML becomes increasingly accessible to biomedical researchers.

\section{Results}

\section{AutoML Model Generation}

We applied an AutoML platform to investigate clinical predictors of SCl patient outcome from intraoperative and acute hospitalization records collected between 2005-2011 and curated by the Transforming Research and Clinical Knowledge for SCI (TRACK-SCI) program, one of the largest SCl patient registries in the US ${ }^{20}$. We selected 46 variables (i.e. features) as predictors from de-identified data of 74 patients (Stable 1). Of these, 16 features were summary statistics (i.e. mean, standard deviation, skew, and kurtosis) derived from timeseries data capturing heart rate, systolic blood pressure, diastolic blood pressure, and mean arterial pressure (MAP) during $\mathrm{SCl}$ surgery. As intraoperative hypertension and hypertension have been shown to be detrimental to $\mathrm{SCl}$ outcome ${ }^{26,27}$, we also calculated the time each patient spent outside of previously-established upper (104 mm Hg) or lower (76 $\mathrm{mmHg}$ ) MAP thresholds during surgery (time_MAP_Avg_above_104 and time_MAP_Avg_below_76, 
respectively $)^{28}$. We defined the prediction target as whether the patient's ASIA Impairment Scale (AIS) score, a common $\mathrm{SCl}$ outcome assessment ${ }^{29}$, improved between time of hospital admission and time of hospital discharge.

To account for potential instability in model optimization, we applied a repeated 10 -fold cross-validation strategy with 25 repetitions where each had a unique partitioning arrangement (i.e. 25 projects) ${ }^{30,31}$. We then aggregated the results for analysis. AutoML generated $80-90$ blueprints: unique combinations of data preprocessing methods and ML algorithms. From these, the platform fully optimized 30-40 models, 15 of which had better mean performance (lower LogLoss and higher AUC) than the benchmark majority class classifier model (Fig. 2A, 2B).

For the purposes of illustration, we selected two high performance blueprints for further interpretation and validation. The first was a $L 2$ regularized logistic regression model with a spline transformation of numeric variables during data preprocessing ( $\left.\mathrm{BP}_{\log } ; \mathrm{Fig} .2 \mathrm{C}\right)$. $\mathrm{BP}_{\log }$ had the best overall performance by LogLoss $(0.67 \pm 0.01)$ and was a top performer by AUC $(0.68 \pm 0.02)$. To apply our framework to a highly complex model use case and given the popularity of XGB in biomedical ML research, we also examined the "eXtreme gradient boosted trees classifier with unsupervised learning features" blueprint with the best LogLoss performance in its class ( $\mathrm{BP}_{\mathrm{XGB}}$; LogLoss $=0.68 \pm 0.01 ; \mathrm{AUC}=0.67 \pm 0.02$ ). Importantly, $\mathrm{XGB}$ trees have gained popularity in biomedical $\mathrm{ML}$ research. $\mathrm{BP}_{\mathrm{XGB}}$ specifically includes a TensorFlow Variational Autoencoder preprocessing $\operatorname{step}^{32}$ (Fig. 2D) as the "unsupervised learning feature", exemplifying the availability of sophisticated methodologies through AutoML platforms.

\section{Feature Importance}

We utilized a permutation-based approach ${ }^{33}$ to quantify feature importance $(\mathrm{pFI})$ for $\mathrm{BP}_{\log }$ and $\mathrm{BP} \mathrm{XGB}_{\mathrm{XG}}$. Notably, pFI values from individual models varied significantly; we accordingly aggregated $\mathrm{pFI}$ across the 25 projects for more robust comparisons. While the order of features by importance for $\mathrm{BP}_{\log }$ and $\mathrm{BP}_{\mathrm{XGB}}$ were different, we observed that many of the high importance features for both models were the timeseries summary statistics (SFig. 1). Interestingly, time_MAP_Avg_above_104 and time_MAP_Avg_below_76 were the most important features for $\mathrm{BP}_{\log }(\mathrm{SFig} .1 \mathrm{~A})$ but were $11^{\text {th }}$ and $18^{\text {th }}$ in rank respectively for $B P_{X G B}$ (SFig. 1B).

\section{Performance Precision}

We observed that different partitioning arrangements resulted in varying model performances even with the same blueprint (Fig. 3A, 3B). We sought to determine whether aggregating the results of 25 projects significantly improved the precision of model performances (i.e. performance precision) towards reproducible comparisons between blueprints. Measures of performance precision, including descriptive statistics such as $95 \%$ confidence intervals $(\mathrm{Cl})$, provide a critical measure of the reliability of the modeling process and is applicable to both AutoML and single model workflows. We additionally contextualized performance precision relative to the mean model performance:

$$
\text { Standardized performance CI width }=\frac{\text { Observed Confidence Interval Width }}{\text { Mean Performance }} * 100
$$


Standardized performance $\mathrm{Cl}$ width provides a measure of model precision in the context of the model's performance; for example, a $\mathrm{Cl}$ width of \pm 0.5 is effectively imprecise if the mean performance value is also 0.5 . Large standardized $\mathrm{Cl}$ width values indicate high unreliability in the modeling process and thus caution against definitively comparing model performances in such cases.

To characterize how performance precision changes with the number of projects aggregated, we ran 150 projects with $\mathrm{BP}_{\log }$ and $\mathrm{BP}_{\mathrm{XGB}}$ and performed a sampling analysis (see Methods). For $\mathrm{BP}_{\log }$, we observed that aggregating 25 projects more than halved the expected standardized performance $\mathrm{Cl}$ width when compared to only 2 projects: by LogLoss, $5.22 \pm 0.24 \%$ vs $1.85 \pm 0.01 \%$ for 2 projects vs 25 projects respectively (Fig. $3 \mathrm{~A}$ ) and by AUC, $8.03 \pm 0.38 \%$ vs $2.79 \pm 0.02 \%$ respectively (SFig. $2 \mathrm{~A}$ ). Analysis of $\mathrm{BP}_{\mathrm{XGB}}$ performance precision generated similar results: by LogLoss, $5.42 \pm 0.32 \%$ vs $2.06 \pm 0.04 \%$ for 2 projects vs 25 projects respectively (Fig. 3B) and by AUC, $8.36 \pm 0.38 \%$ vs $2.84 \pm 0.02 \%$ respectively (SFig. $2 \mathrm{~B})$. The performance precision analysis further highlights the variability in model performances by different partitioning arrangements even if the blueprint and training dataset are unchanged. Running multiple projects and aggregating the results increases performance precision and ultimately improves confidence in model comparisons. From the 25 projects in our primary repeated cross-validation workflow, we observed standardized $\mathrm{Cl}$ widths of $1.92 \%$ by LogLoss and $2.88 \%$ by AUC for $\mathrm{BP}_{\log }$ and $1.55 \%$ by LogLoss and $2.73 \%$ by AUC for $\mathrm{BP}_{\mathrm{XGB}}$.

\section{Feature Instability}

We similarly observed that different partitioning arrangements resulted in pFI variability (i.e. feature instability). Given two different $\mathrm{pFI}$ lists - for example, from two different modeling projects or multiproject aggregates with corresponding averaged $\mathrm{pFI}$ - we can quantify the differences between them by calculating:

$$
\text { Feature Rank Instability }(F R I)=\sum_{i=1}^{f}\left|p F I \operatorname{rank}_{a, i}-p F I \operatorname{rank}_{b, i}\right|,
$$

where $a$ and $b$ represent two different $\mathrm{pFI}$ lists, $i$ is the ith feature, and $f$ is the total number of features. $\mathrm{FRI}$ essentially sums the difference in the ranking of features by $\mathrm{pFI}$ for the features shared between any two pFI lists; higher FRI indicates more feature instability.

Similar to the performance precision analysis, we performed a sampling analysis with 150 projects to determine the relationship between number of projects aggregated and feature instability for $\mathrm{BP}_{\log }$ and $\mathrm{BP}_{\mathrm{XGB}}$ (see Methods). We observed extremely high FRI when the number of aggregated projects is small, suggesting that $\mathrm{pFI}$ can differ significantly from one project to another. When we increased the number of aggregated projects towards 150, FRI decreased towards 0 , indicating that $\mathrm{pFI}$ ranking can be stabilized with sufficient project aggregation. At 25 projects, $\mathrm{BP}_{\log }$ and $\mathrm{BP}_{\mathrm{XGB}}$ had average $\mathrm{FRI}$ values of $13.03 \pm 0.34$ and $11.65 \pm 0.33$ respectively (Fig. 3C, 3D). This approximately amounted to a $93 \%$ decrease in instability for both $\mathrm{BP}_{\log }$ and $\mathrm{BP}_{\mathrm{XGB}}$ as compared to when only aggregating across 2 projects.

\section{Automated Feature Reduction}

A component of AutoML is automating feature reduction (i.e. variable selection) to obtain a more parsimonious feature list ${ }^{34,35}$. This is particularly important for clinical models since clinical features 
often outnumber the observations in biomedical datasets, increasing the danger of model overfitting ${ }^{36}$. We employed an iterative backward wrapper approach utilizing pFI to determine and remove the lowest-importance features at each step (see Methods).

Importantly, we needed to ascertain the stability of features to be eliminated. Because our approach initially removes five features at a time, we accordingly applied our feature instability analysis to just the five lowest-importance features. With 25 projects, we found that $\mathrm{BP}_{\log }$ had a FRI value of $0.96 \pm 0.08$ (Fig. 3E) and $\mathrm{BP}_{\mathrm{XGB}}$ had a FRI value of $0.56 \pm 0.06$ (Fig. 3F) for the bottom five features. In contrast, the bottom five features cumulatively shifted at least eight ranks on average if we aggregated only two projects. By aggregating across 25 projects, we can be confident that the least important features are reliably the lowest ranked.

The best-performing parsimonious $\mathrm{BP}_{\mathrm{log}}$ had an average LogLoss of $0.55 \pm 0.02$ and only nine retained features (Fig. 4A). Of these, the highest pFl features included time_MAP_Avg_above_104, time_MAP_Avg_below_76, and the MRI BASIC score (MRI_1_BASIC_Score), a neuroimaging score for injury severity collected upon hospital admission (Fig. 4B) ${ }^{37}$. The corresponding mean AUC $(0.83 \pm 0.02)$ was also close to the maximum AUC of the feature-reduced models (SFig. 3A).

Interestingly, initial feature reduction with $\mathrm{BP}_{\mathrm{XGB}}$ removed the time_MAP_Avg_below_76 feature. Given that clinical experts and $\mathrm{SCl}$ literature emphasize the correlation between hypotension and worse patient outcome $\mathrm{e}^{28}$, we tested if preserving time_MAP_Avg_below_76 during feature reduction would produce better parsimonious model performance. The resulting parsimonious $\mathrm{BP}_{\mathrm{XGB}}$ model included 11 features, had an average LogLoss of $0.48 \pm 0.02$ (Fig. 4C), and was close to the maximum AUC observed $(0.87 \pm 0.01)$ (SFig. 3B). Notably, this final performance was better than the best parsimonious model when time_MAP_Avg_below_76 had been eliminated (LogLoss $=0.52 \pm 0.02$; AUC $=0.87 \pm 0.01$ ). Furthermore, time_MAP_Avg_below_76 did not end up as the lowest-ranked feature in the final parsimonious feature list despite requiring user guidance to prevent elimination (Fig. 4D). Since time_MAP_Avg_below_76 exhibited collinearity with the other surgical timeseries-derived features, the handling of collinear features by the XGB algorithm is likely why time_MAP_Avg_below_76 was dropped without user intervention. Indeed, we observed higher $\mathrm{FRI}$ at each feature reduction step for $\mathrm{BP} \mathrm{XGB}_{\mathrm{B}}$ as compared to $\mathrm{BP}_{\text {log }}$ corresponding with larger $\mathrm{pFI}$ changes for $\mathrm{BP} \mathrm{XGB}_{\mathrm{B}}$ as collinear features are eliminated (SFig. 4). Additionally of interest, the most important feature of the parsimonious $B P_{X G B}$ feature list was the AIS score at admission (AIS_ad) which provides similar context for initial injury severity as the MRI BASIC score ${ }^{37}$.

\section{Feature Interpretation}

For additional interpretability, we utilized partial dependence plots (PDPs) to quantify the relationship of each individual features' values to the model's prediction ${ }^{38-40}$. PDPs are a model-agnostic approach and thus can be applied regardless of the preprocessing steps or algorithm implemented. We aggregated the partial dependence for each feature for $\mathrm{BP}_{\log }$ and $\mathrm{BP}_{\mathrm{XGB}}$ across the 25 projects (SFig. 5, SFig. 6).

The PDPs of the initial injury severity features - MRI_1_BASIC_Score for BP $\mathrm{log}_{\text {log }}$ and AIS_ad for $\mathrm{BP}_{\mathrm{XGB}}-$ captured nuances between the two (Fig. 5A, 5B). We observed that a BASIC score of 4 , which corresponds to severe injuries with notable hemorrhage, reduced the probability of patient improvement. Similarly, patients classified as AIS A (i.e. complete, severe SCIs) had the lowest 
probability of outcome improvement. Both PDPs thus conveyed that the most severe $\mathrm{SCl}$ cases are unlikely to see improvement by the time of hospital discharge. However, the two scores had different effects for mild injuries: for $\mathrm{BP}_{\mathrm{log}}$, low BASIC scores (0-3) all increased the probability of improvement whereas AIS D reduced the likelihood of improvement for $B P_{X G B}$. This underscores the difference in sensitivity and granularity between the AIS and BASIC scores. AIS D broadly encapsulates mild SCls and is effectively a ceiling on the scale since improvement requires full recovery, which is uncommon. BASIC scores of 0-2 cover a range of functionally mild-to-moderate SCIs; indeed, BASIC and AIS scores do not correlate $1: 1^{37}$. Overall, the results suggested that patients with moderate $\mathrm{SCls}$ (AIS B and C; BASIC 2) have the highest likelihood of outcome improvement.

PDPs of time_MAP_Avg_above_104 and time_MAP_Avg_below_76 revealed that the models predicted worse outcome if a patient exceeded $104 \mathrm{mmHg}$ by more than 70 minutes (Fig. 5C, 5D) or dropped below $76 \mathrm{mmHg}$ for more than 150 minutes (Fig. 5E, 5F). $\mathrm{BP}_{\log }$ and $\mathrm{BP}_{\mathrm{XGB}}$ produced similar time_MAP_Avg_above_104 and time_MAP_Avg_below_76 PDPs, though we observed that $\mathrm{BP} \mathrm{XGB}_{\mathrm{XG}}$ predicted relatively better outcome for patients at the extreme upper range of time ( $>115 \mathrm{~min}$ for time_MAP_Avg_above_104 and $>200$ min for time_MAP_Avg_below_76). This is likely due to the dataset having fewer patients at the extreme ranges rather than a true clinical effect. Critically, $\mathrm{BP}_{\text {log }}$ implemented a spline transformation (Fig. 2C) and quintiled the continuous variables; all patients exceeding 70 or 150 minutes outside the upper or lower thresholds respectively were categorized to the same quintile. Accordingly, $\mathrm{BP}_{\text {log }}$ would not produce the $\mathrm{PDP}$ rebound observed with $\mathrm{BP}_{\mathrm{XGB}}$.

\section{MAP Threshold Validation}

We previously found that time outside the MAP range of $76-104 \mathrm{mmHg}$ was associated with lower probability of AIS improvement as determined by LASSO logistic regression models testing different MAP ranges while expanding the lower and upper MAP thresholds simultaneously ${ }^{28}$. To validate the MAP thresholds, we started with the best-performing parsimonious feature lists, removed the MAP threshold features, and then swept through various lower threshold $(70-85 \mathrm{mmHg}$ ) or upper threshold $(95-115 \mathrm{mmHg})$ features separately to identify how the threshold affected prediction.

With $\mathrm{BP}_{\mathrm{log}}$, we observed the best performances with lower thresholds of $74-76$ or $79 \mathrm{mmHg}$ and with upper thresholds at 103-105 mmHg by LogLoss (Fig. 6A). With $\mathrm{BP}_{\mathrm{XGB}}$, we observed the best

performances at lower thresholds of $74-76 \mathrm{mmHg}$ and at upper thresholds at 103-104 mmHg (Fig. 6B). The results were similarly reflected with AUC (SFig. 7), corroborating the thresholds of 76 and 104 $\mathrm{mmHg}$ for predicting patient outcome. Our analysis furthermore revealed that the time spent above the upper threshold improved the predictive performance of the models significantly more, highlighting intraoperative hypertension as an important correlate and a potential factor for worse $\mathrm{SCl}$ recovery.

\section{Model Validation}

We visualized how each step of the workflow improves AUC and corresponding receiver operating characteristic (ROC) curves. Starting with $\mathrm{BP}_{\mathrm{log}}$ and all our predictors except MAP threshold features, we achieved an average AUC of $0.63 \pm 0.019$ (SFig. 8A). Inclusion of MAP threshold features (with 76 and $104 \mathrm{mmHg}$ thresholds) improved AUC to $0.68 \pm 0.02$ (SFig. 8B), underscoring the importance of intraoperative MAP regulation. The largest improvement to model performance occurred after feature 
reduction to a more parsimonious feature list (AUC $0.84 \pm 0.02$ ) (SFig. 8C). Adjusting the MAP thresholds produced miniscule improvements to AUC (AUC $0.85 \pm 0.02$ ) (SFig. 8D). Lastly, by repeating the process with $\mathrm{BP}_{\mathrm{XGB}}$, prediction performance was improved to AUC $0.87 \pm 0.01$ (SFig. 8E). Altogether, we can improve model performance by adjusting the feature list and model by leveraging the AutoML workflow (SFig. 8F). Importantly, the ROC curves from each of the 25 projects notably differ, emphasizing how varying the dataset partitioning can produce significant model variability.

Critically, the greatest obstacle for translating predictive models into the clinic is the validity of the models on novel data. While many ML scenarios implement a holdout partition from the original dataset for validation, clinical datasets often have relatively small sample sizes where such practices would result in underfitted models, especially for medical fields with smaller patient populations such as $\mathrm{SCl}^{41}$. Clinical model validity is thus often assessed with an external validation dataset collected from a new cohort of patients. Here, we obtained additional data for external validation from a prospective (2015 onward) TRACK-SCl cohort of 59 patients. Of these, 14 patients improved in outcome while the remainder 45 did not.

We used the parsimonious $\mathrm{BP}_{\log }$ and $\mathrm{BP}_{\mathrm{XGB}}$ models to predict the probability of AIS improvement of the validation cohort. We aggregated these values across the 25 projects and generated plots and confusion matrices using the mean predictions and best F1 thresholds calculated by the AutoML platform. The 9feature parsimonious $\mathrm{BP}_{\text {log }}$ model correctly predicted 13 of the 14 patients who improved but only 15 of the 45 patients who did not (Fig. 7A; SFig. 9A). The 11-feature parsimonious $\mathrm{BP}_{\mathrm{XGB}}$ model correctly predicted only 9 of the 14 patients with AIS improvement and 14 of the 45 patients without (Fig. 7B; SFig. 9B). While $\mathrm{BP}_{\mathrm{XGB}}$ has higher predictive accuracy on the training dataset, the model did not perform as well on novel data as the $\mathrm{BP}_{\log }$ model.

We hypothesized that the poor validation performance was due to data drift where the validation patient population no longer resembled that of the training dataset. We accordingly performed population similarity analysis, starting with population stability index (PSI) assessment for each of the parsimonious features. PSI broadly reflects the differences in value distribution between the two cohorts $^{42}$. We observed that most of the features exhibited significant (PSI >0.25) or moderate (PSI > 0.1 ) drift between training and validation datasets, and only TBI_Present and Vertebral_Artery_Injury features could be considered to not have drifted (PSI < 0.1) (STable 2). The PSI results overall suggested that the training and validation populations are dissimilar, thus resulting in poor model performance during validation.

To investigate clinical trends underlying data drift, we clustered all the patients by the raw feature values of the 15 parsimonious features via UMAP and HDB clustering. We observed notable differences in cluster representation: in particular, the validation cohort was only sparsely represented in Clusters 1 and 2 as compared to the training cohort (Fig. 7C). We summarized the distribution of values within each cluster to better understand the subpopulation characteristics and found that Cluster 1 was defined by extremely high Time_to_OR and Cluster 2 by extremely high time_MAP_Avg_below_76 (STable 3). In discussion with clinical experts, we found that this corresponded with shifting clinical practices to reduce time-to-surgery and prevent intraoperative hypotension ${ }^{43,44}$. Population similarity analysis thus provided critical insight into the differences between training and validation populations while demonstrating the crucial need to validate predictive models and corresponding conclusions before translating findings to the clinic. 
Overall, we illustrate a framework to augment AutoML for interpretability, reproducibility, and validity. The process presents opportunities to integrate methods for explainable and reproducible Al that are essential for biological inferences and evidence-based clinical practice. Augmenting AutoML to generate verifiable machine intelligence will be critical to building confidence and trust in powerful Al tools towards advancing biomedical research and precision medicine.

\section{Discussion}

As AutoML become increasingly efficient at optimizing an ever-growing repertoire of data preprocessing methods and algorithms, biomedical researchers will have more opportunities to leverage powerful models to transform their field $d^{4,6,9,45}$. Importantly, there remains a strong, parallel need for better evaluation and interpretation of AutoML-derived models for healthcare where trust in ML requires reproducibly identifying and validating biological mechanisms before translation to patient care ${ }^{46}$. Here, we demonstrate a framework to improve the reproducibility of $M L$ and AutoML workflows by implementing a repeated cross-validation strategy with performance precision and feature instability metrics, actively incorporating domain expertise throughout model optimization and interpretation, and applying population similarity analysis during model validation to better contextualize model generalizability through broader patient population characterization (Box 1; Fig. 1). By incorporating such methods and strategies that emphasize model interpretability and reproducibility, biomedical experts will be empowered to integrate their domain knowledge into ML processes to construct more

Box 1. Key highlights of the framework for reproducible, interpretable AutoML application in biomedicine

- Perform modeling with a repeated cross-validation strategy. Aggregating across models mitigates spurious findings due to model variance from implicit modeling parameters that can lead to model instability with smaller clinical datasets, such as partitioning arrangement.

- Stabilize pFI and PDPs for model interpretation by aggregating repeated crossvalidation models to improve inferential reproducibility.

- Characterize performance precision (metrics: performance $\mathrm{Cl}$; standardized performance $\mathrm{Cl}$ width) for more robust model comparisons. Performance precision analysis can be further applied to achieve a target precision in modeling processes.

- Characterize feature instability (metric: $\mathrm{pFI} \mathrm{Cl}$; feature rank instability) to capture pFI variability. Feature instability analysis can be further applied to stabilize pFI-dependent processes such as feature reduction.

- Integrate domain expertise throughout; combining expert guidance and model-driven feature selection can improve final parsimonious model performance. Any inferences drawn from modeling should be verified with clinical expertise.

- Investigate data drift (i.e. population similarity analysis) between the training and external validation datasets during model validation. PSI and clustering analysis can uncover clinical differences between the cohorts and the underlying evolution of clinical practices that further inform model validation results. 
trustworthy models and build confidence in Al-driven applications in biomedicine.

Repeated cross-validation is an easily applied strategy to reinforce the reproducibility of ML research and has been well-established for handling variation in partitioning ${ }^{47,48}$. Yet despite the focus on confidence intervals, significance, and inference in biomedical research, repeated cross-validation is not commonly implemented by clinical ML studies. This is likely due to the associated computational costs, resulting in clinical $M L$ researchers optimizing and drawing conclusions from a single model instead. We show that different dataset partitions affected model performance and pFI values; the practice of only relying on a single optimization can thus lead to false assumptions that one algorithm is definitively better at prediction or that specific features are definitively more important. Additionally, combining repeated optimizations can help stabilize subsequent processes; 25 projects was the critical aggregation threshold for our backward feature reduction approach such that the bottom 5 features by pFI would be reproducibly ranked the least important. Naturally, selecting the number of repetitions ultimately depends on the dataset, the specific model, and the research question or ML process of interest. Nevertheless, a repeated $k$-fold cross-validation strategy to report confidence intervals, performance precision, and feature instability can help contextualize the reproducibility of results and mitigate spurious conclusions whether applying AutoML or a single model blueprint.

The variability we observed also reflects the concept of underspecification in ML: that even with the same model blueprint and training data, different optimizations can produce divergent solutions ${ }^{49}$. Underspecification highlights how seemingly arbitrary modeling choices - such as implicit modeling parameters and data partitioning - can lead to models with high accuracy on training data that then fail to perform on novel data. While this emphasizes the need to validate models, underspecification also underscores an ongoing demand for additional model evaluation metrics. Repeated cross-validation strategies along with performance precision and feature instability analyses can be applied to characterize and control for underspecification factors of model optimization. This can be further extended to deployment applications: bagging models to account for underspecification can improve model validity, and precision and instability analyses can help estimate the effective number of models for ensembling. Moreover, the predictions of the individual models underlying an ensemble can provide useful context about model precision to users and ultimately enhance trustworthiness and adoption for clinical decision-making.

A major challenge in clinical modeling is obtaining external validation data, especially for conditions where the patient population is relatively small ${ }^{20,41}$. Inconsistent data collection methods and standards further exacerbate the difficulties. Many clinical ML studies consequently produce accurate models that remain unvalidated or are later shown to underperform on novel data ${ }^{50-52}$. We obtained data from a recent patient cohort enrolled in the TRACK-SCI prospective study and observed that despite the high performance of the final $\mathrm{BP}_{\log }$ and $\mathrm{BP}_{\mathrm{XGB}}$ models on training data, both performed poorly during validation. Population similarity analysis by PSI revealed that almost every feature exhibited significant data drift, indicating differences between the two patient cohorts. We reviewed the PSI and cluster analysis results with TRACK-SCI clinicians who validated that the observed changes corresponded with evolving guidelines including moving $\mathrm{SCl}$ patients into surgery sooner and improving blood pressure management to avoid hypotension ${ }^{43,44}$. Indeed, both practices were implemented during the prospective era, and the overarching findings illustrate the fact that biomedical data inevitably shifts with everupdating clinical practices ${ }^{25}$. Furthermore, while all of our data was collected at UCSF, the phenomenon of data drift can apply to data from different medical centers; clinical ML studies commonly utilize data 
from only a single source, and resultant models fail to generalize. Thus beyond simply validating models with novel data, the analytical framework for successful clinical ML application should also contrast the training and validation populations with quantitative metrics alongside domain expertise to identify critical clinical context to inform model validity.

Understanding population similarity can also provide avenues for improving clinical models: a simplistic approach could be to retrain the model with more recent or representative data while balancing dataset size and population similarity. Researchers could also directly incorporate sources of data drift and population dissimilarity into the modeling strategy, such as via cross-hospital validation, to produce the most generalizable models and inferences ${ }^{53}$. A third possibility is to combine data across time and centers to obtain a larger sample size, thus allowing for a holdout partition that better mimics the training dataset. With careful, balanced representation of patients and feature values in training and holdout partitions, researchers could identify common predictive features that are generalizable across a broader clinical population. Specific to $\mathrm{SCl}$, harmonizing data across multiple clinical programs could help reinforce future ML studies with a more comprehensive patient dataset. Most importantly, once a model is deployed into clinic, monitoring for data drift will be necessary to determine whether the model will need to be retrained to keep up with changing medical practices.

Especially for datasets with a small observations-to-features ratio as is common in clinical research, parsimony can improve performance by decreasing multicollinearity and removing low-signal features while improving interpretability ${ }^{54}$. We deployed an iterative wrapper approach ${ }^{35}:$ backward feature $^{2}$ selection based on recalculated $\mathrm{pFI}$ rankings to optimize LogLoss. While the process is model-agnostic, the results are affected by the characteristics of the underlying algorithms; the final parsimonious feature lists differed between $\mathrm{BP}_{\text {log }}$ and $\mathrm{BP}_{\mathrm{XGB}}$, and $\mathrm{BP}_{\mathrm{XGB}}$ exhibited in greater $\mathrm{pFl}$ instability between each feature reduction step. This can be attributed to how regularized regression and tree-based models handle multicollinearity - a common trait of clinical datasets - which affects $\mathrm{pFI}$ as colinear features are eliminated. Critically, there is no definitive approach to feature selection; clinical verification of the final parsimonious feature list is necessary given that the preserved features depend heavily on the implemented reduction method and models ${ }^{34,35}$. More broadly, pFI has been shown to overestimate the importance of colinear features, especially for tree-based models ${ }^{55}$; future work should refine the framework by employing techniques to address feature collinearity, such as Shapley additive explanations or accumulated local effects plots in place of $\mathrm{pFI}$ and PDPs respectively ${ }^{56,57}$.

We allowed for preselection of features by domain experts during feature reduction, and because the process inherently optimizes model accuracy, we can determine if preservation of expert-selected features improves or undermines the final model performance. Here, by preserving the time a patient spent below $76 \mathrm{mmHg}$ MAP, we improved the maximum performance for the parsimonious $\mathrm{BP}_{\mathrm{XGB}}$ model. The process can also be applied to test other hypotheses, including ones where deliberate experimentation might be difficult or impossible. For example, whether a $\mathrm{SCl}$ patient receives specific treatments is a matter of clinical care rather than experimental design, but we can compare the impact of excluding or including the treatment feature on the final model accuracy to glean a relationship between treatment and outcome. Such findings would provide further insight to the efficacy of clinical practices that may be difficult to test experimentally and highlight areas for future, targeted clinical research. Importantly, removed variables are not necessarily unimportant or uninformative; feature selection ultimately reflects the representation of samples and the limitations of the dataset. Continual validation and updating of the parsimonious feature list alongside the model are critical for maintaining 
and improving clinical models. This aligns with the budding concept of expert-augmented Al wherein the interaction between human expertise and ML leads to better models.

Our results also provide more granularity on how intraoperative MAP thresholds relate to outcome: the time patients spent outside the upper MAP threshold contributed more to model prediction than the time spent below the lower threshold. Additionally, the corresponding PDPs reveal that the critical outof-threshold duration for worse outcome is shorter for hypertension ( $>70 \mathrm{~min}$ ) than for hypotension ( $>$ $\sim 150 \mathrm{~min}$ ). Previous blood pressure management studies have primarily focused on hypotension as a contributor to worse patient outcome $e^{58-60}$ despite the increased risk of cardiovascular and cerebrovascular complications as a result of hypertension ${ }^{61}$. Notably, the importance of perioperative hypertension for $\mathrm{SCl}$ outcome has been observed both clinically and preclinically ${ }^{26-28,62}$; this is the first analysis to suggest that hypertension is more predictive of worse outcome than hypotension, thus proposing that careful MAP management should strive to avoid hypertension while minimizing hypotension. Future prospective clinical studies should extend the verifiability of the findings throughout broader $\mathrm{SCl}$ patient care such as during treatment in the emergency room and intensive care unit. Moreover, the illustrated framework can be similarly applied to investigate other modifiable components of clinical care as well as identify predictors of other patient outcome metrics including chronic recovery.

Ultimately, there remains significant untapped potential for Al-driven impact on clinical practices, precision medicine, and general healthcare. While not completely unique to biomedicine, the challenges of achieving inferential reproducibility from relatively small sample sizes dictate the need for parallel development of explainable and reproducible Al methods that augment powerful processes such as AutoML. By unboxing even the most complex models and accounting for the uncertainty associated with ML, we can build an essential culture of trust for Al with biomedical researchers and clinicians towards a bidirectional relationship where clinicians actively guide Al development and Al applications effectively support clinical decision-making to improve patient care.

\section{Methodology}

\section{Datasets}

The data were collected and de-identified by the Transforming Research and Clinical Knowledge for Spinal Cord Injury (TRACK-SCI) program ${ }^{20}$ and contains clinical variables (i.e. features) collected during acute hospitalization and $\mathrm{SCl}$-related surgery. The training dataset consisted of 74 clinical records collected between 2005-2011. After implementing our AutoML workflow, we obtained a second dataset for model validation from TRACK-SCI consisting of 59 clinical records collected after 2015.

Of note, 18 of the 47 features in the full feature list were derived from time-series data for intraoperative heart rate, systolic blood pressure, diastolic blood pressure, and mean arterial pressure (MAP). Each set of time-series data was summarized as mean, standard deviation, skew, and kurtosis features for each individual patient. Additionally, the total time each patient spent above or below a MAP threshold (starting with 104 and $76 \mathrm{mmHg}$ respectively) was derived from the time-series MAP data. The prediction target AIS_is_improved was derived from the patients' AIS scores as assessed by clinicians using the International Standards for Neurological Classification of SCI (ISNCSCI) exam at the 
time of hospital admission and discharge. Specifically, AIS_is_improved was assigned a value of 0 for no improvement or a value of 1 if the patient's AIS score improved between admission (AIS_ad) and discharge (AIS_dis). Notably, the training data included 39 patients who improved in AIS score while the validation dataset included 14 such patients. The AIS_dis feature was excluded for model training since it was used to derive the prediction target and would cause target leakage. The remaining 46 features used for modeling are listed in Supplementary Table 1.

\section{AutoML Model Generation and Feature Importance}

Among various available implementations of AutoML, we utilized the DataRobot commercial AutoML platform for our workflow ${ }^{5}$. Access and application of the platform was done primarily through the API in Python. Performance values (LogLoss and Area Under Curve; AUC), mutual information between predictors, permutation feature importance $(\mathrm{pFI})$, partial dependence plot (PDP) values, receiver operating characteristic (ROC) values, model validation predictions, estimated best F1 thresholds, and population stability index (PSI) values were downloaded and then analyzed and graphed in $\mathrm{R}$ with the tidyverse package $e^{63,64}$.

The training data was uploaded to the platform which generates a new project instance (i.e. project). Each project encapsulates a specific set of modeling inputs and parameters such as the training data, the type of ML problem (i.e. regression vs classification), partitioning strategy, and others that define the initial state of the AutoML process. For our projects, we assigned distinct random seeds which specifically affected the unique partitioning arrangement of the data for modeling. Given that the AutoML platform requires a minimum of 100 observations to perform automated classification modeling, we accounted for the small number of records in our dataset by duplicating the entry for each patient, thus doubling the dataset from 74 to 148 records. Importantly, we specified a 10 -fold crossvalidation strategy while ensuring that each of the ten partitions had at least one representation of each of the possible prediction target values and that duplicated records were always partitioned together.

For the first round of modeling, we included 46 features as predictors (Supplementary Table 1). The AutoML platform generated 80-90 possible configurations of data preprocessing steps and algorithms (i.e. blueprints, with each blueprint being assigned a unique identification number). Blueprints range from simple (e.g. $\mathrm{BP}_{\text {log: }}$ regularized logistic regression model with a spline transformation preprocessing step) to complex (e.g. $\mathrm{BP}_{\mathrm{XGB}}$ : extreme gradient boosted tree with a modified TensorFlow Variational Autoencoder preprocessing step), and the platform automatically performs data preprocessing and algorithm-specific optimization to maximize the final model performance. To identify the bestperforming models, the platform first trained the blueprints on a small subset of the dataset and selected the top performing blueprints according to their validation LogLoss accuracy. The blueprint selection process was repeated with a larger subset of data for a second round of selection. The remaining blueprints, numbering between 30-40 total, were then optimized on the full dataset, and cross-validation accuracy was calculated.

To characterize the stability of the modeling process, we applied the strategy of repeated 10-fold crossvalidation with 25 repetitions. Each repetition corresponded to a project with a unique random seed that determined the unique arrangement of data in the partitions. Each project implemented the same blueprints for AutoML; we accordingly aggregated the performances for each blueprint across all 25 
projects by calculating the mean and $95 \%$ confidence interval for the corresponding cross-validation performances (i.e. performance). We arranged the models according to mean performance and plotted those that outperformed the Majority Class Classifier benchmark model which simply predicts every patient as having improved - the majority class of the AIS_is_improved target.

Permutation feature importance ( $\mathrm{pFl}$; also termed feature impact on the platform) was calculated through a permutation-based approach by the AutoML platform ${ }^{33}$. In brief, the values of a single feature were permuted, and the resulting loss in LogLoss accuracy was calculated. The permutation and performance loss assessment were repeated multiple times to generate an average accuracy loss. This process was performed on every feature individually. The platform further normalized the pFI values to the maximum $\mathrm{pFI}$ value observed; $\mathrm{pFI}$ in this study thus refers to the normalized values. We aggregated the $\mathrm{pFI}$ values across all 25 projects to calculate the mean $\mathrm{pFI}$ and $95 \%$ confidence interval for each feature. The features were then arranged from highest to lowest pFI for visualization.

\section{Performance Precision Analysis}

To characterize the relationship between number of aggregated projects and the precision (i.e. variability) of model performance as a result of different partitioning arrangements, we created 150 projects, each with a unique random seed and corresponding partitioning arrangement. We optimized both $\mathrm{BP}_{\log }$ and $\mathrm{BP}_{\mathrm{XGB}}$ in each project with the 46 features using the AutoML platform. We collected the resulting cross-validation LogLoss and AUC performance values for all 150 projects. We then performed the following sampling analysis:

1. Randomly sample one project.

2. Randomly sample another project without replacement. The newly-sampled project and any previously-sampled projects form the current project aggregate.

3. Calculate the standardized performance $\mathrm{Cl}$ width with the current project aggregate.

4. Repeat steps 2-3 until all 150 projects have been aggregated.

5. Perform steps 1-4 1000 times.

6. Calculate the expected (i.e. mean) standardized performance $\mathrm{Cl}$ width with corresponding $95 \%$ confidence intervals for each number-of-projects-aggregated.

Standardized performance $\mathrm{Cl}$ width was calculated with the following formula:

$$
\text { Standardized performance CI width }=\frac{\text { Observed Confidence Interval Width }}{\text { Mean Performance }} * 100
$$

The results were visualized with an emphasis on the 25 -project point. The process was repeated for $\mathrm{BP}_{\log }$ and $\mathrm{BP} \mathrm{XGB}_{\mathrm{X}}$ as well as for LogLoss and AUC metrics.

\section{Feature Instability Analysis}

To characterize the relationship between number of aggregated projects and feature instability, we used the same 150 projects as in the performance precision analysis. For each instance of $\mathrm{BP}_{\mathrm{log}}$ and $\mathrm{BP}_{\mathrm{XGB}}$, we calculated the normalized $\mathrm{pFI}$ values. To obtain the $\mathrm{pFI}$ ranks, we ordered and ranked the $\mathrm{pFI}$ values for each specific instance of the model and project from highest to lowest. As a metric for feature instability, we calculated the feature rank instability (FRI): 


$$
\text { Feature Rank Instability }(F R I)=\sum_{i=1}^{f}\left|F I \operatorname{rank}_{p, i}-F I \operatorname{rank}_{q, i}\right|,
$$

where $p$ and $q$ represent two different $\mathrm{pFI}$ lists, $i$ is the ith feature, and $f$ is the total number of features.

We then performed a sampling analysis as follows:

1. Randomly sample one project.

2. Randomly sample another project without replacement. The newly sampled project and any previously sampled projects form the current project aggregate.

3. Rank the features according to the mean $\mathrm{pFI}$ values in the current project aggregate.

4. Calculate FRI between the $\mathrm{pFI}$ lists from the previous project aggregate and the current project aggregate. If only two projects have been aggregated, FRI is calculated between the $\mathrm{pFI}$ list from the first sampled project and the $\mathrm{pFl}$ list from the current project aggregate.

5. Repeat steps 2-3 until all 150 projects have been aggregated.

6. Perform steps 1-5 1000 times.

7. Calculate the expected (i.e. mean) FRI with corresponding $95 \%$ confidence intervals for each number-of-projects-aggregated.

The results were visualized with an emphasis on the 25-project point.

We also performed the sampling analysis on just the ranking of the bottom five features by pFI (i.e. least important features). In this case, the entire feature list was ranked as before, but the FRI was only calculated for the five least important features based on the aggregate with fewer projects (i.e. when comparing 3-project aggregate vs 4-project aggregate, we considered the bottom 5 features from the $\mathrm{pFI}$ values of the 3-project aggregate).

To investigate the feature instability during feature reduction for $\mathrm{BP}_{\log }$ and $\mathrm{BP} \mathrm{XGB}_{\mathrm{B}}$, we applied the $\mathrm{FRI}$ quantification to compare the feature list before and after each reduction step. Specifically, we calculated FRI for the features that remained after elimination. For example, at feature list size $=41$, we calculate the FRI for the 41 features by comparing their rankings between the 46-feature model (before reduction) and the 41-feature model (after reduction).

\section{Automated Feature Reduction}

We applied an iterative wrapper feature reduction process implementing backward elimination similar to as historically applied to regression models ${ }^{34}$. Notably, the lowest-ranking features by pFI were removed; this feature reduction process can be applied to any blueprint on the AutoML platform. The process is as follows:

1. Start with the full feature list.

2. Calculate average $\mathrm{pFI}$ values for each feature across the 25 projects.

3. Remove the 5 features with lowest mean $\mathrm{pFI}$ values.

4. Optimize new models on remaining features.

5. Repeat steps 2-4 until no features remain.

6. Identify the range of feature list sizes containing the likely maximum performance.

7. Repeat steps 2-4 within the range identified in step 6 and using a step size of 1 . 
The initial step size of five was chosen to balance for computational time needed to retrain 25 models at each elimination step. By aggregating across the 25 projects, we were able to stabilize the pFI rankings.

Importantly, identifying the final parsimonious feature list was determined directly by the resulting model performances. At each elimination step, the model cross-validation performance was calculated and averaged across projects for comparison. The mean model performance values were used to pinpoint the feature list size range at step 6 as well as identify the final best-performing parsimonious model and feature list.

To test whether preservation of time_MAP_Avg_below_76 would improve final parsimonious model performance with $\mathrm{BP}_{\mathrm{XGB}}$, we allowed users to preselect features that the process would never eliminate (equivalent to augmenting feature reduction with expert guidance). If the preselected features landed in the elimination range of the $\mathrm{pFI}$ ranking, the process selected the next lowest-importance feature instead. We accordingly selected time_MAP_Avg_below_76 to be preserved.

\section{Feature Interpretation}

The AutoML platform implements partial dependence plots (PDPs) for feature interpretation ${ }^{40}$. In brief, the platform averaged the outcome predictions for the training dataset while converting the values of a single feature to a single value. The set value for the feature of interest was then changed, scanning either across the continuous range or all possible categorical values depending on the feature's data type. Plotting the average outcome prediction by the possible feature values produced the feature's PDP for the model. We additionally pooled the partial dependence values across the 25 projects, calculated the mean and $95 \%$ confidence intervals, and created an aggregated PDP for each feature in the parsimonious $\mathrm{BP}_{\log }$ and $\mathrm{BP}_{\mathrm{XGB}}$ models.

\section{MAP Threshold Validation}

To investigate the MAP thresholds that would be most predictive of patient outcome, we first removed the MAP threshold features from the final parsimonious feature lists of $\mathrm{BP}_{\log }$ and $\mathrm{BP} \mathrm{XGB}_{\mathrm{XG}}$. We then created new lists by including a single MAP threshold feature using a different lower (in range of $70-85 \mathrm{mmHg}$ ) or upper (in range of 95-115 mmHg) threshold. Sweeping through each possible threshold value, this produced 16 feature lists with a lower MAP threshold feature and 21 feature lists with an upper MAP threshold feature. We additionally included a feature list with no MAP threshold feature. Across the 25 projects, we optimized models for each feature list, aggregated the model performance values, and summarized and plotted the results as mean and $95 \%$ confidence intervals. The model performance for the feature lists including both a lower and upper MAP threshold feature was the resulting parsimonious model from the feature reduction process prior.

\section{Model Validation}

To validate the parsimonious $\mathrm{BP}_{\log }$ and $\mathrm{BP}_{\mathrm{XGB}}$ models, we uploaded the validation dataset to the AutoML platform and predicted the probability of AIS improvement for each patient. The AutoML platform also calculated the best F1 threshold - the value that maximizes the F1 score - for each model in each project. We aggregated the predictions for each patient across the 25 projects to calculate mean and 
$95 \%$ confidence intervals. We similarly summarized the best $\mathrm{F} 1$ threshold values. To produce the confusion matrices, we compared the mean prediction value for each patient against the mean best F1 threshold value. Mean prediction values above the mean F1 threshold were considered positive predictions (i.e. patient improved) and conversely for negative predictions (i.e. no improvement).

To determine whether there is data drift between the training and validation dataset, we deployed the parsimonious models on the DataRobot servers to access the data drift feature. In brief, the platform determines data drift between training and validation datasets by calculating the population stability index (PSI) for each of the features ${ }^{42}$.

Combining both the training and validation datasets, we additionally performed dimensionality reduction via UMAP (umap $\mathrm{R}$ package ${ }^{65}$ ) for the 15 features preserved in the parsimonious $\mathrm{BP}_{\text {log }}$ and $\mathrm{BP}_{\mathrm{XGB}}$ models. Importantly, 9 of the 133 samples were missing values and were thus removed via listwise deletion for clustering analysis prior to UMAP. The resulting UMAP scores were used to cluster the patients via HDB Clustering ( $d b s c a n$ R packag ${ }^{66}$ ) with a minimum cluster size of 8 . The datapoints were then grouped according to training vs validation dataset and plotted. The circular borders containing the clusters were drawn manually for visual clarity. For the numeric features, we calculated the mean and $95 \%$ confidence interval of the distribution within each cluster.

\section{Acknowledgements}

This work was supported by research grants: DoD/CDMRP/SCIRP SC150198 (MSB); DoD/CDMRP/SCIRP SC190233 (MSB); Craig H. Neilsen Foundation SCl - Center of Excellence Award (MSB); NIH/NINDS R01NS088475 (ARF); NIH/NINDS UH3NS106899 (ARF); US Department of Veterans Affairs 1101RX002245 (ARF); US Department of Veterans Affairs I01RX002787 (ARF); Craig H. Neilsen Foundation (ARF); Wings for Life Foundation (ARF); NRSA post-doctoral fellowship NIH/NINDS F32NS117728 (AC).

\section{Competing interests}

SK, JS, JH, AL, RS, and CM are current employees of DataRobot and own shares of the company. Access to the DataRobot Automated Machine Learning platform was awarded through application and selection by the DataRobot Al for Good program. DataRobot affiliated authors provided editorial contributions during the preparation of the manuscript. All other authors declare no competing financial interests. 


\section{Figure Legends}

Figure 1. A framework for applying Automated Machine Learning (AutoML) for reproducible inferences in biomedical research. After data is curated, we perform a cyclical model development process utilizing AutoML to optimize an array of models. Reproducible and explainable Al tools and strategies can be applied to ultimately draw clinical and biological inferences from the models and allow for integration of domain expertise. Critically for clinical modeling, we also include a feature reduction component to achieve a more parsimonious model. The final models are then validated with external validation data along with population similarity analysis for further clinical contextualization. By applying this framework, models produced by AutoML can be stabilized and interpreted for inferential reproducibility and clinical verifiability.

Figure 2. AutoML generated 15 models that performed better than the Majority Class Classifier model by (A) LogLoss and (B) Area Under Curve (AUC). Each model consisted of automatically implemented preprocessing steps and algorithms. Models were assigned names according to the algorithm and encoded by a unique color. Blueprints of the same algorithm class are numbered for identification across both LogLoss and AUC plots. Two models were selected for additional analysis: $\mathrm{BP}_{\mathrm{log}}$ (blue box) and $\mathrm{BP} \mathrm{XGB}_{\mathrm{X}}$ (green box). Aggregating across 25 projects (unique partitioning arrangements of the dataset), $\mathrm{BP}_{\log }$ had an average performance of $0.67 \pm 0.01$ LogLoss and $0.68 \pm 0.02 \mathrm{AUC}$; $\mathrm{BP}_{\mathrm{XGB}}$ had an average performance of $0.68 \pm 0.01$ LogLoss and $0.67 \pm 0.02 \mathrm{AUC}$. (C) $\mathrm{BP}_{\log }$ consisted of a regularized logistic regression (L2) algorithm with a notable quintile spline transformation preprocessing step for numeric variables. (D) $\mathrm{BP}_{\mathrm{XGB}}$ implemented an eXtreme Gradient Boosted (XGB) trees classifier with unsupervised learning features, which refers to the TensorFlow Variational Autoencoder preprocessing step for categorical variables.

Figure 3. Analysis of performance precision and feature instability as a function of number of projects aggregated. Each project uses a unique partitioning arrangement of the dataset. Performance precision is calculated as the standardized performance confidence interval $(\mathrm{Cl})$ width, which scales the $\mathrm{Cl}$ width by the mean performance. Feature instability is calculated as the cumulative shift in feature importance ranking (feature rank instability; FRI). (A, B) Expected values and corresponding confidence intervals of the standardized performance $\mathrm{Cl}$ width. As the number of projects increased, the performance precision improved (i.e. standardized performance $\mathrm{Cl}$ width decreased). By LogLoss, $\mathrm{BP}_{\log }$ started with a performance precision of $5.22 \pm 0.24 \%$ with 2-project aggregation and decreased to an average of $1.85 \pm$ $0.01 \%$ with 25-project aggregation (A). Similarly, $\mathrm{BP} \mathrm{XGB}_{\mathrm{XB}}$ started with a performance precision of $5.42 \pm$ $0.32 \%$ and decreased to an average of $2.06 \pm 0.04 \%$ at 25 projects (B). (C, D) Expected values and corresponding confidence intervals of FRI for all 46 features. As the number of projects increased, FRI decreased (i.e. pFI ranking became more stable). $\mathrm{BP}_{\text {log }}$ had an average FRI of $174.40 \pm 2.14$ with 2-project aggregation and $13.03 \pm 0.34$ with 25-project aggregation $(C)$. Similarly, $B P_{X G B}$ started with an average FRI of $153.83 \pm 3.06$ that decreased to $11.65 \pm 0.33$ at 25 projects (D). (E, F) Focusing only on the bottom five features by $\mathrm{pFI}$ to calculate $\mathrm{FRI}, \mathrm{BP}_{\mathrm{log}}$ had an average $\mathrm{FRI}$ of $20.41 \pm 0.75$ with 2-project aggregation and decreased to $0.96 \pm 0.08$ with 25-project aggregation (E). Similarly, BP ${ }_{\mathrm{XGB}}$ started with an average FRI of $7.77 \pm 0.37$ and decreased to $0.56 \pm 0.06$ for the bottom five features with 25 -project aggregation (F). 
Figure 4. Applying an iterative backward feature reduction process to identify parsimonious feature lists that maximize model performance. The process was performed first by removing the lowest five features by feature importance (step size $=5$ ) and then repeated with step size $=1$ with in the feature list size range that contained the best performance. (A) For $\mathrm{BP}_{\text {log }}$, the step size was reduced starting at 16 features with the best performance observed with the 9-feature parsimonious feature list (LogLoss = $0.55 \pm 0.02$ ). (B) The corresponding $\mathrm{pFI}$ of the 9-feature parsimonious $\mathrm{BP}_{\mathrm{Iog}}$ model showed that the MRI BASIC score and the time patients spent outside of the MAP thresholds were the most important features. The remaining features included other intraoperative timeseries-derived features and the time between hospitalization and surgery (Time_to_OR_a). (C) The feature reduction for $\mathrm{BP} \mathrm{XGB}_{\mathrm{B}}$ was expanded to always preserve the two MAP threshold features. The step size was reduced to one starting at 16 features with the best performance observed with the 11-feature parsimonious feature list (LogLoss = $0.48 \pm 0.02$ ). (D) The corresponding $\mathrm{pFI}$ for the parsimonious $\mathrm{BP}_{\mathrm{XGB}}$ model showed that the AIS score at admission (AIS_ad) was the most important feature. Non-timeseries-derived features included Cervical_Injury, Vertebral_Artery_Injury, and TBI_Present. The time_MAP_Avg_above_104 and time_MAP_Avg_below_76 features were ranked $7^{\text {th }}$ and $9^{\text {th }}$ respectively.

Figure 5. Partial dependence plots (PDPs) for features of interest help interpret how features affect model prediction of $\mathrm{BP}_{\mathrm{log}}$ and $\mathrm{BP}_{\mathrm{XGB}}$. (A) For $\mathrm{BP}_{\mathrm{log}}$, an MRI $\mathrm{BASIC}$ score of 4 resulted in lower prediction of improved outcome. A MRI BASIC score of 0-3 increased prediction of better outcome with a MRI BASIC score of 2 leading to the highest probability of improvement. (B) For $B P_{X G B}$, an AIS score of $A$ or $D$ at admission resulted in lower probability of patient improvement. AIS scores of $B$ and $C$ both led to higher probability of improvement with AIS score $C$ resulting in the highest probability. (C) For $\mathrm{BP}_{\log }$ and (D) $\mathrm{BP}_{\mathrm{XGB}}$, if a patient's MAP exceeded an upper threshold of $104 \mathrm{mmHg}$ for more than 50-75 minutes, the predicted probability of improvement decreased significantly. (E) For $\mathrm{BP}_{\log }$ and (F) $\mathrm{BP} \mathrm{XGB}_{\mathrm{XB}}$, if a patient's MAP fell below a lower threshold of $76 \mathrm{mmHg}$ for more than 100-150 minutes, the predicted probability of improvement decreased significantly. Notably, $\mathrm{BP}_{\mathrm{XGB}}$ PDP for both time_MAP_Avg_above_104 and time_MAP_Avg_below_76 exhibited a rebound in predicted improvement probability at extreme upper values that was absent on the $\mathrm{BP}_{\log }$ PDPs.

Figure 6. LogLoss performance plots for investigating different lower and upper MAP thresholds using best-performing parsimonious $\mathrm{BP}_{\log }$ and $\mathrm{BP}_{\mathrm{XGB}}$ models. (A) With $\mathrm{BP}_{\log }$, we observe that the lower threshold values of $74,75,76$, and $79 \mathrm{mmHg}$ performed the best of the lower thresholds. The upper threshold values of 103, 104, and $105 \mathrm{mmHg}$ performed the best of the upper thresholds. Notably, the best-performing upper threshold feature $(104 \mathrm{mmHg})$ resulted in a larger improvement to model performance compared to the best-performing lower threshold feature $\left(79 \mathrm{mmHg}\right.$ ). (B) With $\mathrm{BP}_{\mathrm{XGB}}$, the values of 74, 75, and $76 \mathrm{mmHg}$ performed the best of the lower thresholds, and the values of 103 and 104 performed the best of the upper thresholds. Similar to $\mathrm{BP}_{\mathrm{log}}$, the best-performing upper threshold feature $(104 \mathrm{mmHg}$ ) resulted in a larger improvement to model performance compared to the bestperforming lower threshold feature $(76 \mathrm{mmHg})$. 
Figure 7. Model validation confusion matrices and clustering analysis to demonstrate differences in patient population between training and validation datasets. Validation predictions were scored by comparing the average predicted probability of each validation sample against the average best F1 threshold for the corresponding model. (A) The best parsimonious $\mathrm{BP}_{\log }$ model correctly predicted 13 of the 14 true positives (i.e. patient improved in outcome) and 15 of the 45 true negatives. (B) The best parsimonious $\mathrm{BP}_{\mathrm{XGB}}$ model correctly predicted 9 of the 14 true positives and 14 of the 45 true negatives. (C) UMAP and HDB clustering analysis on the combined training and validation data produced six clusters of patients. Notably, Clusters 1 and 2 showed high representation in the training cohort and low representation in the validation cohort. Conversely, Cluster 3 showed low and high representation in the training and validation cohorts respectively. Clusters 3, 5, and 6 have no discernable differences between cohorts.

Supplementary Table 1. Features and definitions for the 46 features used for modeling.

Supplementary Figure 1. Normalized permutation feature importance (pFI) of each feature, aggregated

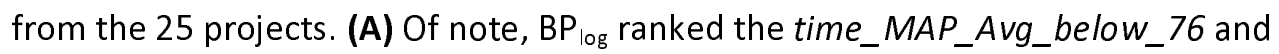
time_MAP_Avg_above_104 highest. (B) Conversely, the two MAP threshold-related features were ranked $11^{\text {th }}$ and $18^{\text {th }}$ in $\mathrm{pFI}$ by $\mathrm{BP}_{\mathrm{XGB}}$. The majority of high $\mathrm{pFI}$ features across both models were features derived from the intraoperative timeseries data for heart rate, diastolic blood pressure, systolic blood pressure, and mean arterial pressure (MAP). Both models also highly ranked a feature encoding initial injury severity: $M R I \_1 \_B A S I C \_S c o r e$ for $\mathrm{BP}_{\log }$ and $A I S \_a d$ for $\mathrm{BP} \mathrm{XGB}_{\mathrm{B}}$.

Supplementary Figure 2. Analysis of performance precision based on AUC performance metric. As observed for performance precision with LogLoss, increasing the number of aggregated projects improved performance precision (i.e. decreased the standardized performance $\mathrm{Cl}$ width). (A) By AUC, $\mathrm{BP}_{\text {log }}$ started with a performance precision of $8.03 \pm 0.38 \%$ and decreased to an average of $2.79 \pm 0.02 \%$ when aggregating 25 projects. (B) $\mathrm{BP}_{\mathrm{XGB}}$ started with a performance precision of $8.36 \pm 0.38 \%$ and decreased to an average of $2.84 \pm 0.02 \%$ when aggregating 25 projects.

Supplementary Figure 3. AUC performances for the feature reduction process. (A) Feature reduction of $\mathrm{BP}_{\log }$ showed maximum $\mathrm{AUC}$ at the 8-feature parsimonious feature list ( $\mathrm{AUC}=0.84 \pm 0.02$ ). The 9feature parsimonious feature list had an $A U C$ of $0.83 \pm 0.02$. (B) Feature reduction of $B P_{X G B}$ showed maximum AUC at the 9-feature parsimonious feature list ( $A U C=0.87 \pm 0.01$ ). The 11-feature parsimonious feature list had a similar AUC of $0.87 \pm 0.01$.

Supplementary Figure 4. Feature instability analysis for $(A) \mathrm{BP}_{\log }$ and $(\mathrm{B}) \mathrm{BP}_{\mathrm{XGB}}$ during backward feature reduction process. FRI was calculated by comparing the $\mathrm{pFI}$ ranking before and after each feature reduction step and only summing the features that appeared in both lists (i.e. features that were not 
removed at the step). Notably, $\mathrm{BP}_{\mathrm{XGB}}$ exhibited higher $\mathrm{FRI}$ at each step than for $\mathrm{BP}_{\text {log; }}$ elimination of features resulted in more shifting of features by $\mathrm{pFI}$ rank for $\mathrm{BP}_{\mathrm{XGB}}$.

Supplementary Figure 5. Partial dependent plots (PDPs) for additional features from the best-

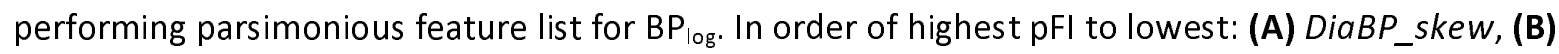
HR_kurtosis, (C) SysBP_sd, (D) MAP_kurtosis, (E) HR_sd, and (F) Time_to_OR_a. PDPs of MRI_1_BASIC_Score, time_MAP_Avg_above_104, and time_MAP_Avg_below_76 are shown in Figure 4.

Supplementary Figure 6. Partial dependent plots (PDPs) for additional features from the bestperforming parsimonious feature list for $\mathrm{BP}_{\mathrm{XGB}}$. In order of highest $\mathrm{pFl}$ to lowest: (A) MAP_kurtosis, (B) DiaBP_skew, (C) HR_sd, (D) Cervical_Injury, (E) TBI_Present, (F) HR_mean, (G) Vertebral_Artery_Injury, and (H) MAP_mean. PDPs of AIS_ad, time_MAP_Avg_above_104, and time_MAP_Avg_below_76 are shown in Figure 4.

Supplementary Figure 7. AUC performance plots for investigating lower and upper MAP thresholds using best-performing parsimonious $\mathrm{BP}_{\log }$ and $\mathrm{BP}_{\mathrm{XGB}}$ models. (A) Similar to the LogLoss plots, the bestperforming lower threshold values were $74,75,76$, and $79 \mathrm{mmHg}$ and the best-performing upper threshold values were 103, 104, and $105 \mathrm{mmHg}$ for $\mathrm{BP}_{\mathrm{log}}$. Of the best-performing thresholds, inclusion of an upper threshold features produced greater improvement to AUC than inclusion of an individual lower threshold feature. (B) For $\mathrm{BP}_{\mathrm{XGB}}$, the best-performing lower threshold values were 74,75 , and $76 \mathrm{mmHg}$, and the best-performing upper threshold values were 103 and $104 \mathrm{mmHg}$. Similar to $\mathrm{BP}_{\text {log, }}$ of the bestperforming thresholds, inclusion of an individual upper threshold feature improved AUC performance more than inclusion of an individual lower threshold feature.

Supplementary Figure 8. Receiver operating characteristic $(R O C)$ curves of individual projects and the averaged curve showing improvement in prediction performance through the workflow. (A) ROC curves of the $L 2$ regularized linear regression model $B_{\text {log }}$ trained on the initial feature list with the exclusion of the MAP threshold features. The average model AUC was $0.63 \pm 0.02$. (B) ROC curves of $\mathrm{BP}_{\log }$ trained on the full feature list including the two MAP threshold features. The average AUC was $0.68 \pm 0.02$. (C) ROC curves after performing feature reduction with $\mathrm{BP}_{\text {log }}$ to find the best-performing parsimonious model (9feature parsimonious feature list). The average AUC increased to $0.84 \pm 0.02$. (D) ROC curves after testing different MAP thresholds with $\mathrm{BP}_{\text {log }}$ and selecting for the best-performing lower $(79 \mathrm{mmHg})$ and upper (104 mmHg) thresholds. The resulting AUC improved incrementally (AUC $0.85 \pm 0.02$ ) compared to using $76 \mathrm{mmHg}$ and $104 \mathrm{mmHg}$. (E) ROC curves after performing the workflow on the eXtreme gradient boosted tree model $\mathrm{BP}_{\mathrm{XGB}}$. The parsimonious feature list consisted of 11 features and the bestperforming MAP thresholds were 76 and $104 \mathrm{mmHg}$. The average model AUC was $0.87 \pm 0.01$. 
Supplementary Figure 9. Model validation plots from performing predictions across the 25 projects with a validation cohort of 59 patients. Of these, 14 patients improved in AIS score while 45 patients did not. Best F1 thresholds as calculated by the AutoML platform were also aggregated from each project (shown in red). (A) Prediction for each validation subject by $\mathrm{BP}_{\text {log }}$. The average best $\mathrm{F} 1$ threshold is $0.41 \pm$ 0.04. (B) Prediction for each validation subject by $B P_{X G B}$. The average best $F 1$ threshold is $0.46 \pm 0.04$.

Supplementary Table 2. Population stability index (PSI) of the parsimonious $\mathrm{BP}_{\log }$ and $\mathrm{BP}_{\mathrm{XGB}}$ model features.

Supplementary Table 3. Within-cluster mean and $95 \%$ confidence interval of numeric features. 


\section{References}

1. Yao, Q. et al. Taking Human out of Learning Applications: A Survey on Automated Machine Learning. ArXiv181013306 Cs Stat (2019).

2. Escalante, H. J., Montes, M., Sucar, L. E., Mx, I. \& Mx, I. Particle Swarm Model Selection. 36.

3. Feurer, M. et al. Efficient and Robust Automated Machine Learning. 9.

4. Balaji, A. \& Allen, A. Benchmarking Automatic Machine Learning Frameworks. ArXiv180806492 Cs Stat (2018).

5. Muhlestein, W. E. et al. Using a Guided Machine Learning Ensemble Model to Predict Discharge Disposition following Meningioma Resection. J. Neurol. Surg. Part B Skull Base 79, 123-130 (2018).

6. Das, P. et al. Amazon SageMaker Autopilot: a white box AutoML solution at scale. ArXiv201208483 Cs (2020).

7. Zöller, M.-A. \& Huber, M. F. Benchmark and Survey of Automated Machine Learning Frameworks. ArXiv190412054 Cs Stat (2020).

8. Waring, J., Lindvall, C. \& Umeton, R. Automated machine learning: Review of the state-of-the-art and opportunities for healthcare. Artif. Intell. Med. 104, 101822 (2020).

9. LeDell, E. \& Poirier, S. H2O AutoML: Scalable Automatic Machine Learning. 16.

10. Kourou, K., Exarchos, T. P., Exarchos, K. P., Karamouzis, M. V. \& Fotiadis, D. I. Machine learning applications in cancer prognosis and prediction. Comput. Struct. Biotechnol. J. 13, 8-17 (2015).

11. Chaikijurajai, T., Laffin, L. J. \& Tang, W. H. W. Artificial Intelligence and Hypertension: Recent Advances and Future Outlook. Am. J. Hypertens. 33, 967-974 (2020).

12. Inoue, T. et al. XGBoost, a Machine Learning Method, Predicts Neurological Recovery in Patients with Cervical Spinal Cord Injury. Neurotrauma Rep. 1, 8-16 (2020).

13. Yu, M. K. et al. Visible Machine Learning for Biomedicine. Cell 173, 1562-1565 (2018).

14. Torkamani, A., Andersen, K. G., Steinhubl, S. R. \& Topol, E. J. High Definition Medicine. Cell 170, 828-843 (2017).

15. Goodman, S. N., Fanelli, D. \& Ioannidis, J. P. A. What does research reproducibility mean? Sci. Transl. Med. 8, 341ps12-341ps12 (2016).

16. Roberts, M. et al. Common pitfalls and recommendations for using machine learning to detect and prognosticate for COVID-19 using chest radiographs and CT scans. Nat. Mach. Intell. 3, 199-217 (2021).

17. Shaikhina, T. \& Khovanova, N. A. Handling limited datasets with neural networks in medical applications: A small-data approach. Artif. Intell. Med. 75, 51-63 (2017).

18. Vabalas, A., Gowen, E., Poliakoff, E. \& Casson, A. J. Machine learning algorithm validation with a limited sample size. PLOS ONE 14, e0224365 (2019). 
19. National Spinal Cord Injury Statistical Center, Facts and Figures at a Glance. Birmingham, AL: University of Alabama at Birmingham (2019).

20. Tsolinas, R. E. et al. Transforming Research and Clinical Knowledge in Spinal Cord Injury (TRACKSCI): an overview of initial enrollment and demographics. Neurosurg. Focus 48, E6 (2020).

21. Kyritsis, N. et al. Diagnostic blood RNA profiles for human acute spinal cord injury. J. Exp. Med. 218, (2021).

22. McCoy, D. B. et al. Convolutional Neural Network-Based Automated Segmentation of the Spinal Cord and Contusion Injury: Deep Learning Biomarker Correlates of Motor Impairment in Acute Spinal Cord Injury. Am. J. Neuroradiol. 40, 737-744 (2019).

23. Chang, M., Canseco, J. A., Nicholson, K. J., Patel, N. \& Vaccaro, A. R. The Role of Machine Learning in Spine Surgery: The Future Is Now. Front. Surg. 7, (2020).

24. Khan, O. et al. Predictive Modeling of Outcomes After Traumatic and Nontraumatic Spinal Cord Injury Using Machine Learning: Review of Current Progress and Future Directions. Neurospine 16, 678685 (2019).

25. Kelly, C. J., Karthikesalingam, A., Suleyman, M., Corrado, G. \& King, D. Key challenges for delivering clinical impact with artificial intelligence. BMC Med. 17, 195 (2019).

26. Ehsanian, R. et al. Exploration of surgical blood pressure management and expected motor recovery in individuals with traumatic spinal cord injury. Spinal Cord 58, 377-386 (2020).

27. Nielson, J. L. et al. Topological data analysis for discovery in preclinical spinal cord injury and traumatic brain injury. Nat. Commun. 6, 8581 (2015).

28. Torres Espín, A. et al. Topological network analysis of patient similarity for precision management of acute blood pressure in spinal cord injury. elife (2021). (In submission)

29. van Middendorp, J. J. et al. Diagnosis and Prognosis of Traumatic Spinal Cord Injury. Glob. Spine J. 1, 1-8 (2011).

30. Krstajic, D., Buturovic, L. J., Leahy, D. E. \& Thomas, S. Cross-validation pitfalls when selecting and assessing regression and classification models. J. Cheminformatics 6, 10 (2014).

31. BURMAN, P. A comparative study of ordinary cross-validation, v-fold cross-validation and the repeated learning-testing methods. Biometrika 76, 503-514 (1989).

32. Kingma, D. P. \& Welling, M. An Introduction to Variational Autoencoders. Found. Trends ${ }^{\circledR}$ Mach. Learn. 12, 307-392 (2019).

33. Breiman, L. Random Forests. Mach. Learn. 45, 5-32 (2001).

34. Sanchez-Pinto, L. N., Venable, L. R., Fahrenbach, J. \& Churpek, M. M. Comparison of Variable Selection Methods for Clinical Predictive Modeling. Int. J. Med. Inf. 116, 10-17 (2018).

35. Bagherzadeh-Khiabani, F. et al. A tutorial on variable selection for clinical prediction models: feature selection methods in data mining could improve the results. J. Clin. Epidemiol. 71, 76-85 (2016). 
36. Subramanian, J. \& Simon, R. Overfitting in prediction models - Is it a problem only in high dimensions? Contemp. Clin. Trials 36, 636-641 (2013).

37. Talbott, J. F. et al. The Brain and Spinal Injury Center score: a novel, simple, and reproducible method for assessing the severity of acute cervical spinal cord injury with axial T2-weighted MRI findings. J. Neurosurg. Spine 23, 495-504 (2015).

38. Goldstein, A., Kapelner, A., Bleich, J. \& Pitkin, E. Peeking Inside the Black Box: Visualizing Statistical Learning with Plots of Individual Conditional Expectation. ArXiv13096392 Stat (2014).

39. Molnar, C. Interpretable Machine Learning. https://christophm.github.io/interpretable-mlbook/\# (2020).

40. Friedman, J. H. Greedy function approximation: A gradient boosting machine. Ann. Stat. 29, 1189-1232 (2001).

41. DeVivo, M. J. Epidemiology of traumatic spinal cord injury: trends and future implications. Spinal Cord 50, 365-372 (2012).

42. Karakoulas, G. Empirical validation of retail credit-scoring models. 5.

43. Fehlings, M. G. et al. A Clinical Practice Guideline for the Management of Acute Spinal Cord Injury: Introduction, Rationale, and Scope. Glob. Spine J. 7, 84S-94S (2017).

44. Harrop, J. S. et al. Controversies in Spinal Trauma and Evolution of Care. Neurosurgery 80, S23S32 (2017).

45. Golovin, D. et al. Google Vizier: A Service for Black-Box Optimization. in Proceedings of the 23rd ACM SIGKDD International Conference on Knowledge Discovery and Data Mining 1487-1495 (ACM, 2017). doi:10.1145/3097983.3098043.

46. Richens, J. G., Lee, C. M. \& Johri, S. Improving the accuracy of medical diagnosis with causal machine learning. Nat. Commun. 11, 3923 (2020).

47. Jr, F. E. H. Regression Modeling Strategies: With Applications to Linear Models, Logistic and Ordinal Regression, and Survival Analysis. (Springer, 2015).

48. de Rooij, M. \& Weeda, W. Cross-Validation: A Method Every Psychologist Should Know. Adv. Methods Pract. Psychol. Sci. 3, 248-263 (2020).

49. D'Amour, A. et al. Underspecification Presents Challenges for Credibility in Modern Machine Learning. ArXiv201103395 Cs Stat (2020).

50. Kappen, T. H. et al. Evaluating the impact of prediction models: lessons learned, challenges, and recommendations. Diagn. Progn. Res. 2, 11 (2018).

51. Steyerberg, E. W. et al. Prognosis Research Strategy (PROGRESS) 3: Prognostic Model Research. PLOS Med. 10, e1001381 (2013).

52. Bleeker, S. E. et al. External validation is necessary in prediction research:: A clinical example. J. Clin. Epidemiol. 56, 826-832 (2003). 
53. Bolourani, S. et al. Development and Validation of a Machine learning prediction model of respiratory failure within 48 hours of patient admission for COVID-19. J. Med. Internet Res. (2021) doi:10.2196/24246.

54. Saeys, Y., Inza, I. \& Larranaga, P. A review of feature selection techniques in bioinformatics. Bioinformatics 23, 2507-2517 (2007).

55. Hooker, G. \& Mentch, L. Please Stop Permuting Features: An Explanation and Alternatives. ArXiv190503151 Cs Stat (2019).

56. Lundberg, S. M. \& Lee, S.-I. A Unified Approach to Interpreting Model Predictions. Adv. Neural Inf. Process. Syst. 30, (2017).

57. Apley, D. Visualizing the Effects of Predictor Variables in Black Box Supervised Learning Models. (2016).

58. Vale, F. L., Burns, J., Jackson, A. B. \& Hadley, M. N. Combined medical and surgical treatment after acute spinal cord injury: results of a prospective pilot study to assess the merits of aggressive medical resuscitation and blood pressure management. J. Neurosurg. 87, 239-246 (1997).

59. Levi, L., Wolf, A. \& Belzberg, H. Hemodynamic parameters in patients with acute cervical cord trauma: description, intervention, and prediction of outcome. Neurosurgery 33, 1007-1016; discussion 1016-1017 (1993).

60. Hawryluk, G. et al. Mean Arterial Blood Pressure Correlates with Neurological Recovery after Human Spinal Cord Injury: Analysis of High Frequency Physiologic Data. J. Neurotrauma 32, 1958-1967 (2015).

61. Aronow, W. S. Management of hypertension in patients undergoing surgery. Ann. Transl. Med. 5, (2017).

62. Almeida, C. A. et al. Excavating FAIR Data: the Case of the Multicenter Animal Spinal Cord Injury Study (MASCIS), Blood Pressure, and Neuro-Recovery. Neuroinformatics (2021) doi:10.1007/s12021021-09512-z.

63. R Core Team. R: A Language and Environment for Statistical Computing. (R Foundation for Statistical Computing, 2020).

64. Wickham, H. et al. Welcome to the \{tidyverse\}. J. Open Source Softw. 4, 1686 (2019).

65. Konopka, T. umap: Uniform Manifold Approximation and Projection. (2020).

66. Hahsler, M., Piekenbrock, M. \& Doran, D. dbscan: Fast Density-Based Clustering with R. J. Stat. Softw. 91, 1-30 (2019). 


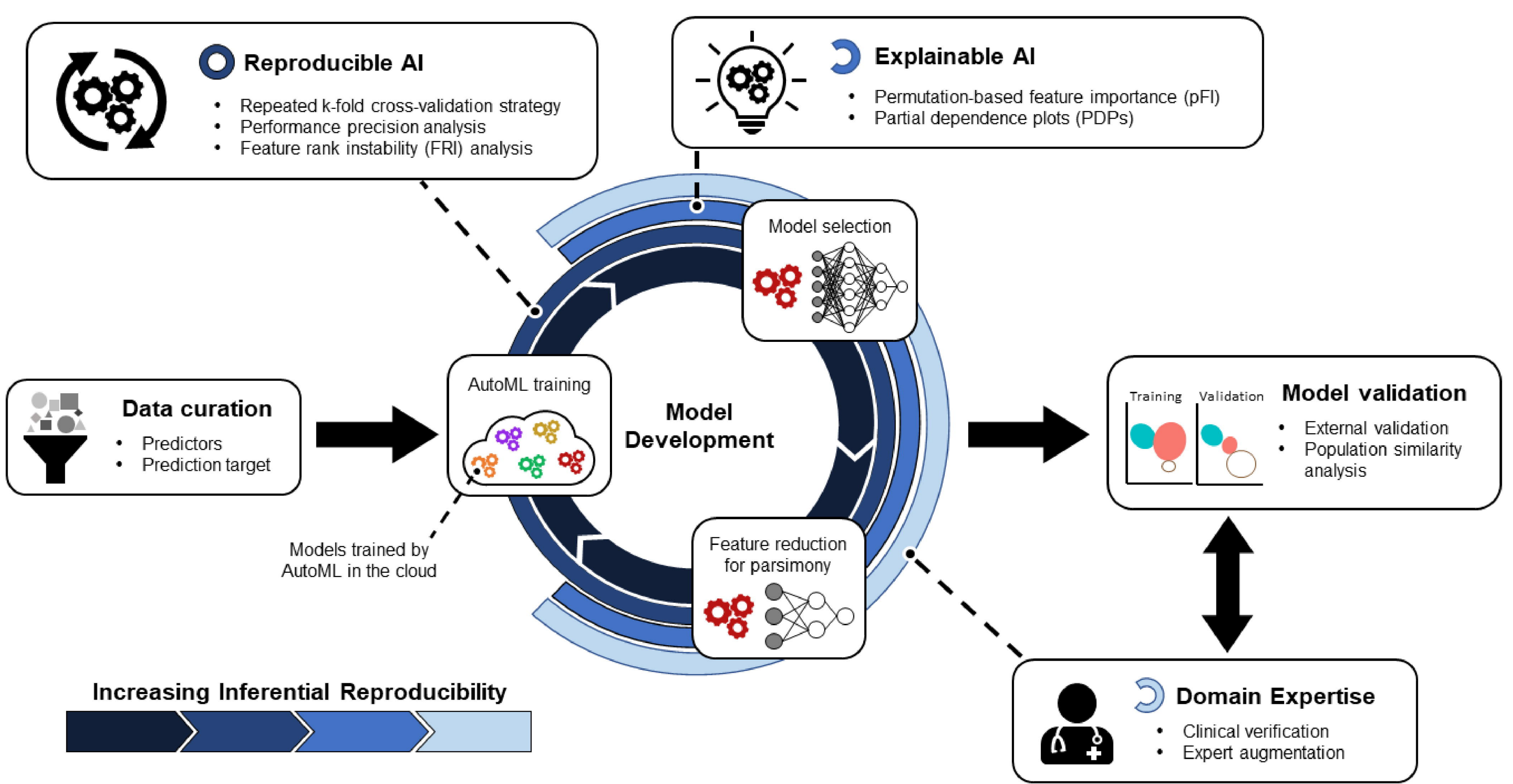




\section{Figure 2}

A

$$
\text { A }
$$

Regularized Logistic Regression $\left(\mathrm{BP}_{\mathrm{log}}\right)$
Breiman and Cutler Random Forest

Light Gradient Boosted Trees

Gradient Boosted Trees (1)

XGB

GB with Unsupervised Learning Features (BP $\left.\mathrm{XGB}_{\mathrm{B}}\right)$

XGB with Unsupervised Learning Features (2)

XGB with Unsupervised Learning Features (3)

Gradient Boosted Greedy Trees

RandomForest (Entropy)

RandomForest (Gini) (1)

Regularized Logistic Regression (2)

Vowpal Wabbit

Gradient Boosted Trees (2)

Regularized Logistic Regression (3)

Majority Class Classifier

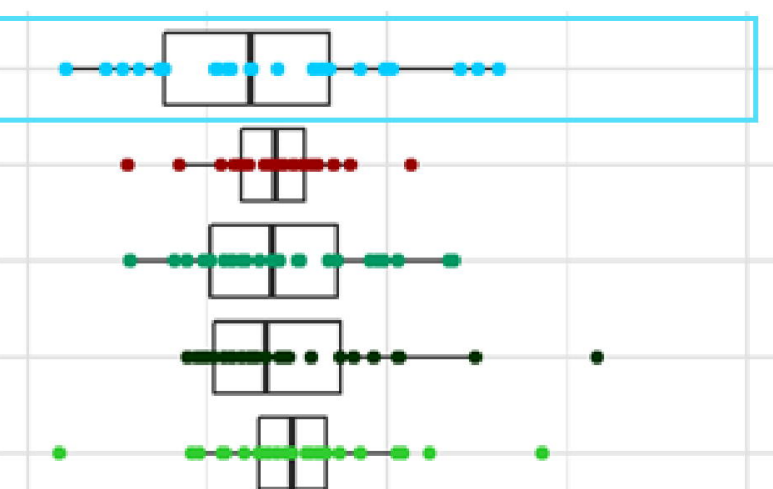

17

)

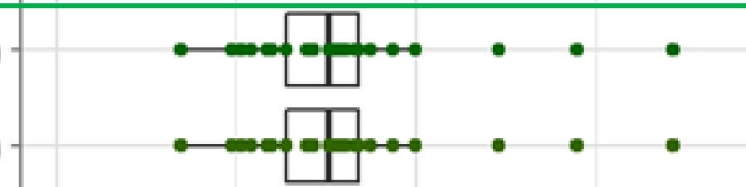

$\frac{11}{11}$

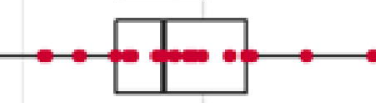

ता

U
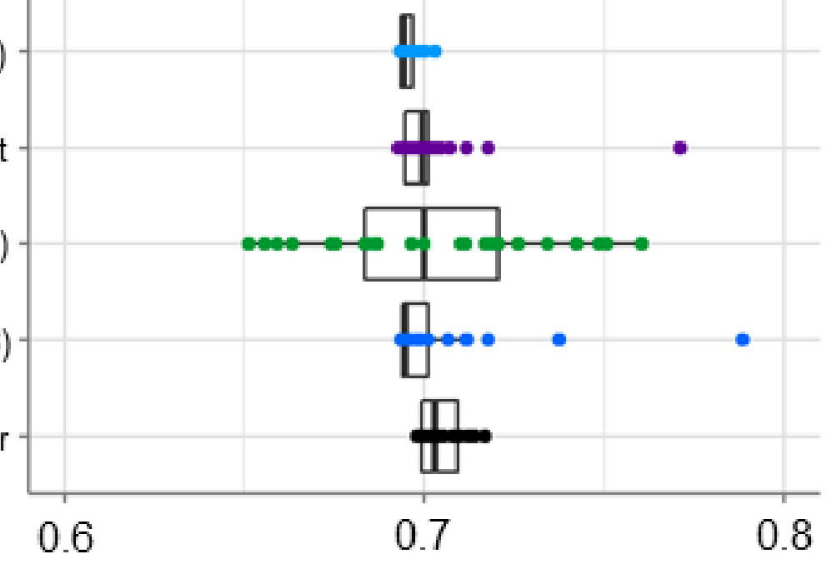

B

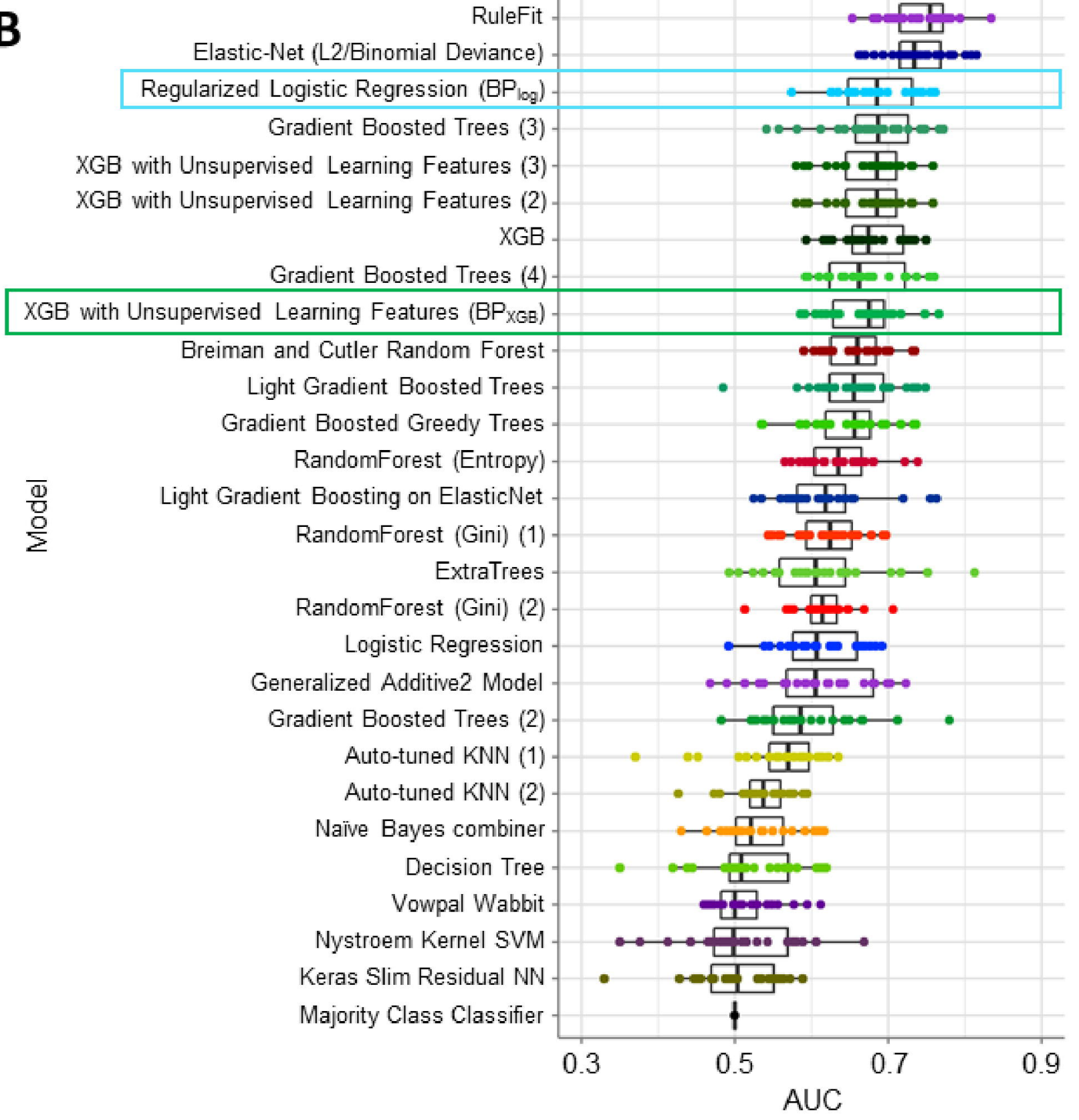

C Blueprint of Best-Performance Regularized Logistic Regression Model $\left(\mathrm{BP}_{\log }\right)$

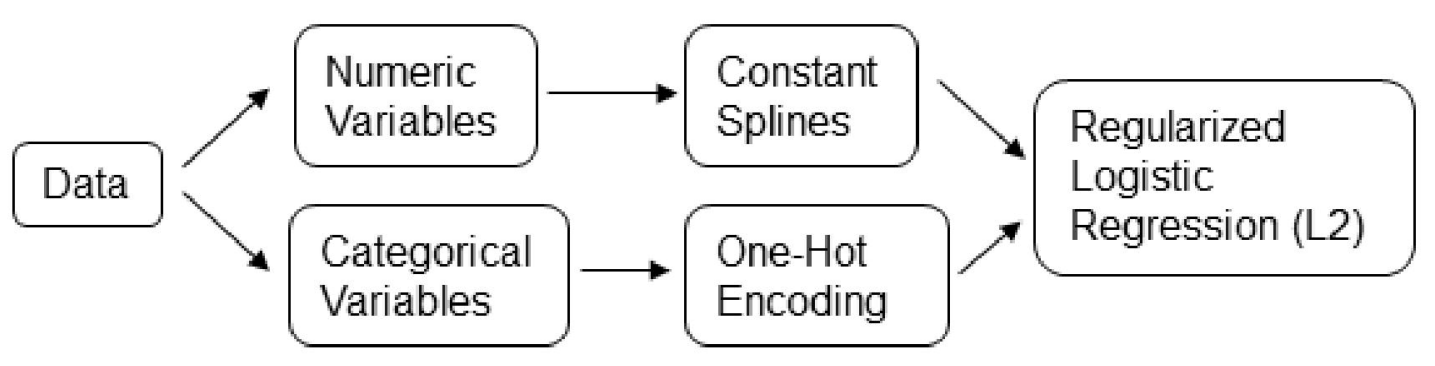

D Blueprint of Best-Performance eXtreme Gradient Boosted Trees Classifier with Unsupervised Learning Features Model $\left(\mathrm{BP} \mathrm{XGB}_{\mathrm{B}}\right)$

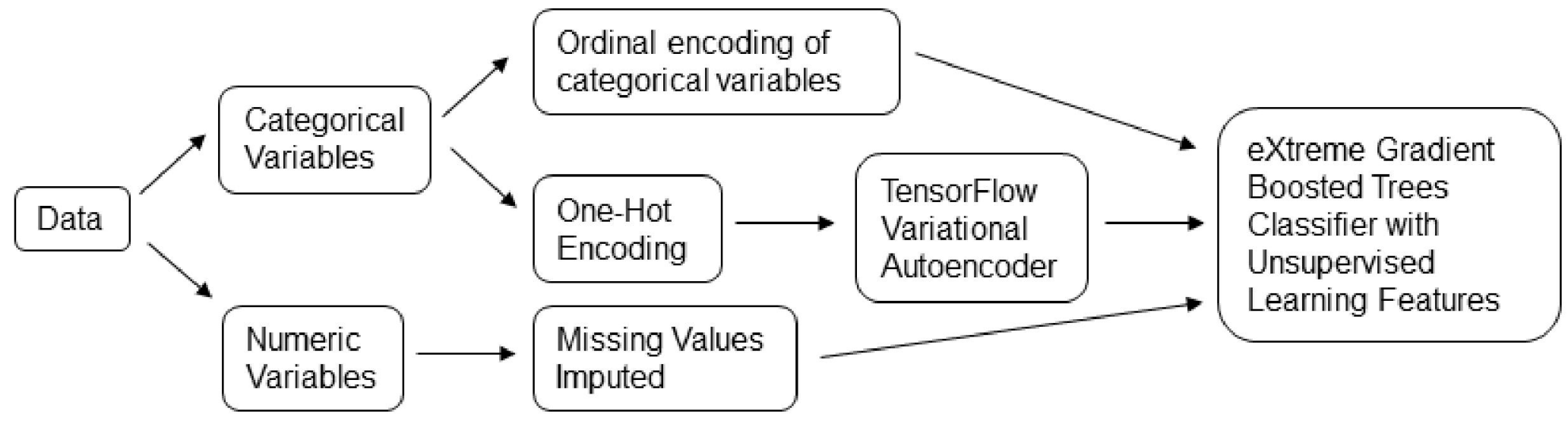




\section{Figure 4}

A $\mathrm{BP}_{\text {log: }}$ LogLoss

B $\quad \mathrm{BP}_{\mathrm{log}}$, feature list size $=9$
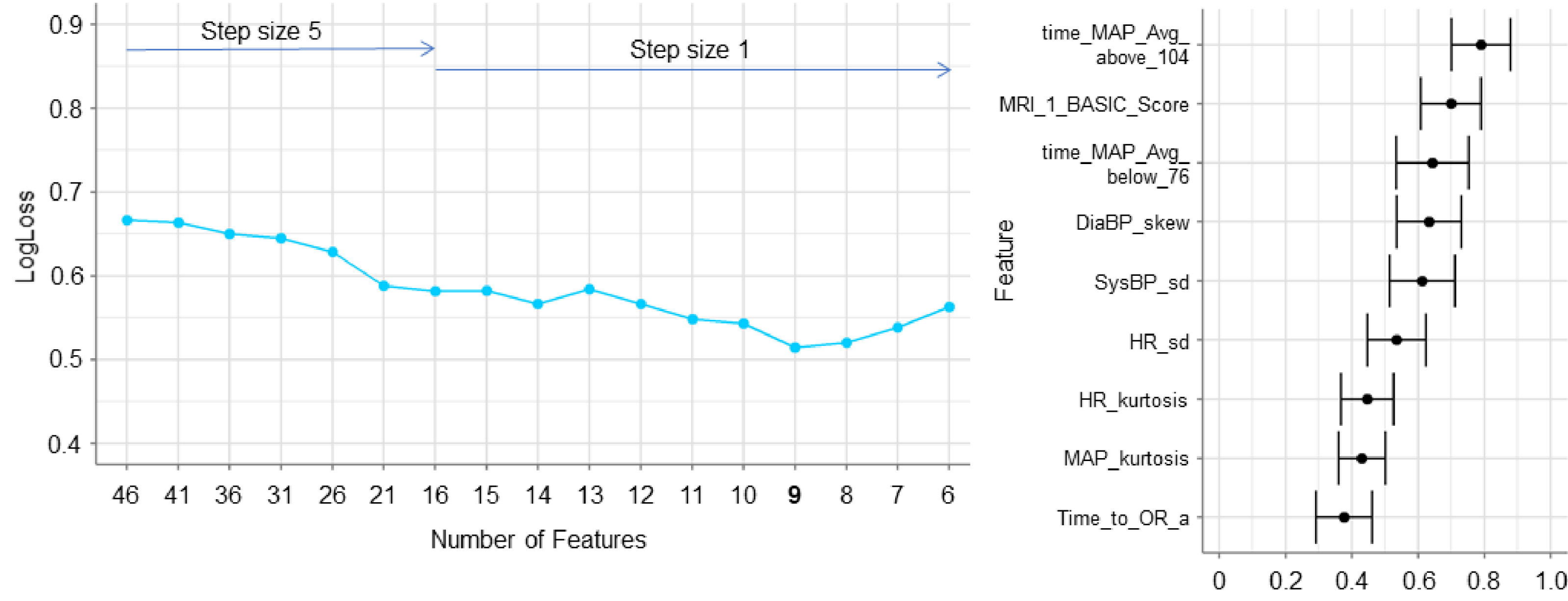

Normalized Feature Importance

C BP $_{\mathrm{XGB}}$ : LogLoss

D BP $_{\mathrm{XGE}}$, feature list size $=11$
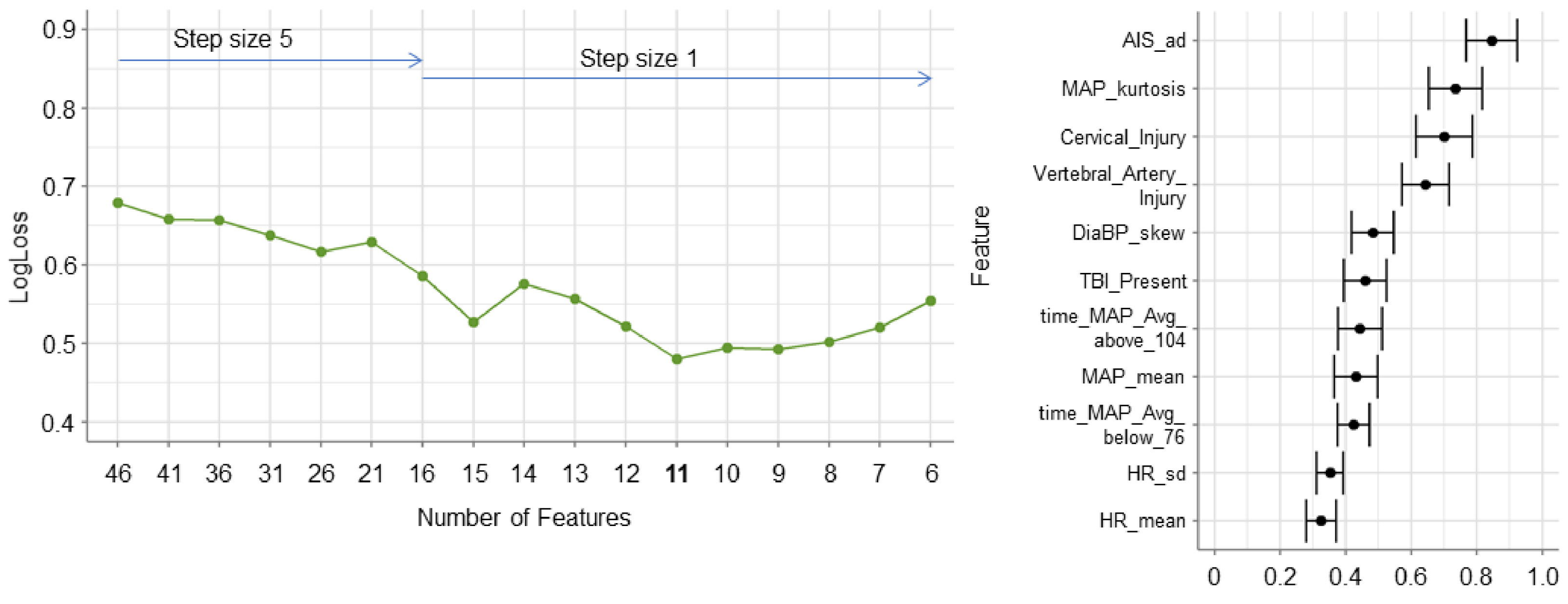

Normalized Feature Importance 
Figure 5

A BP $\mathrm{Blog}_{\mathrm{O}}: M R I$ 1_BASIC_Score

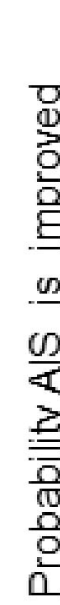

0.7 .

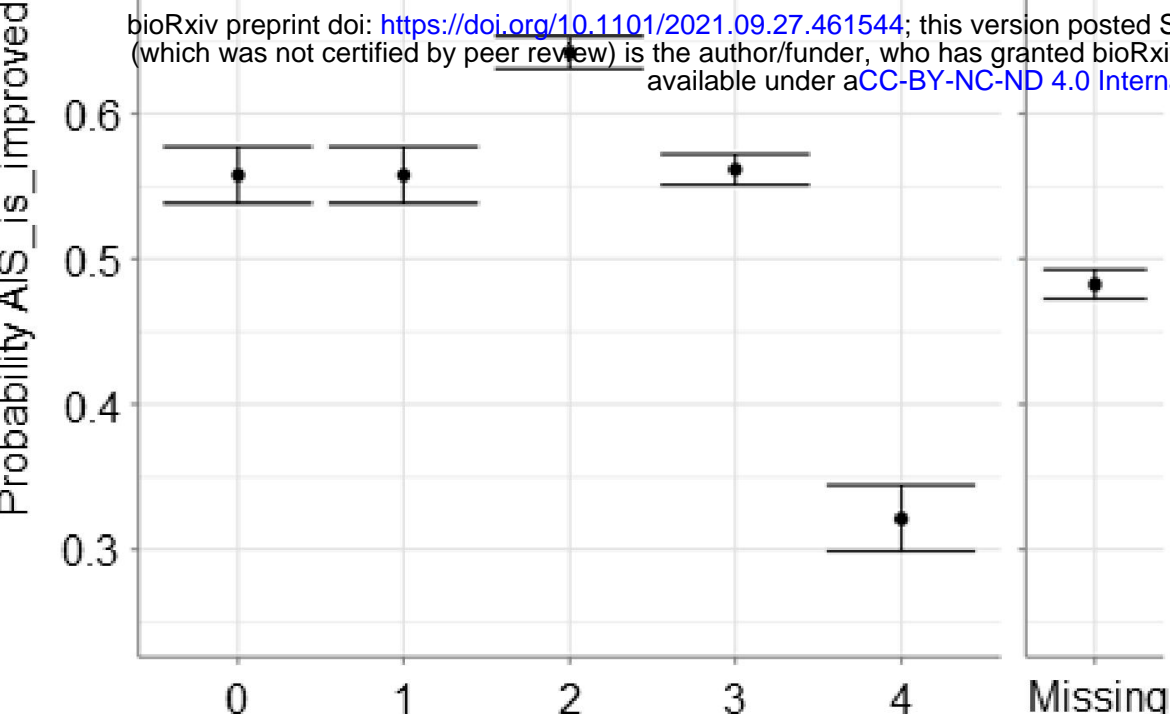

MRI BASIC Score

C BP log: time_MAP_above_104

(1)

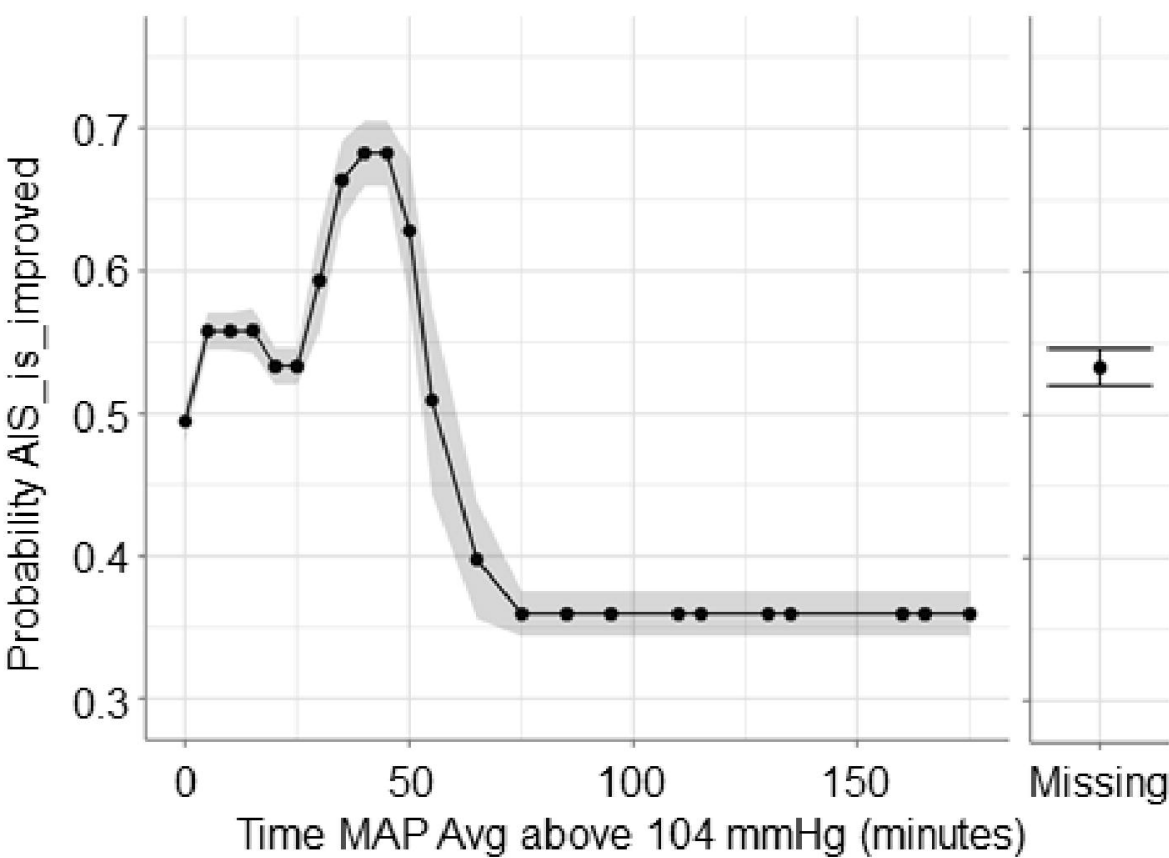

E $\quad \mathrm{BP}_{\text {log: }}$ - time_MAP_below_76

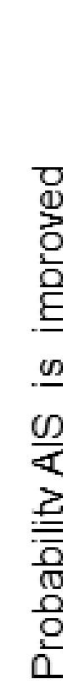

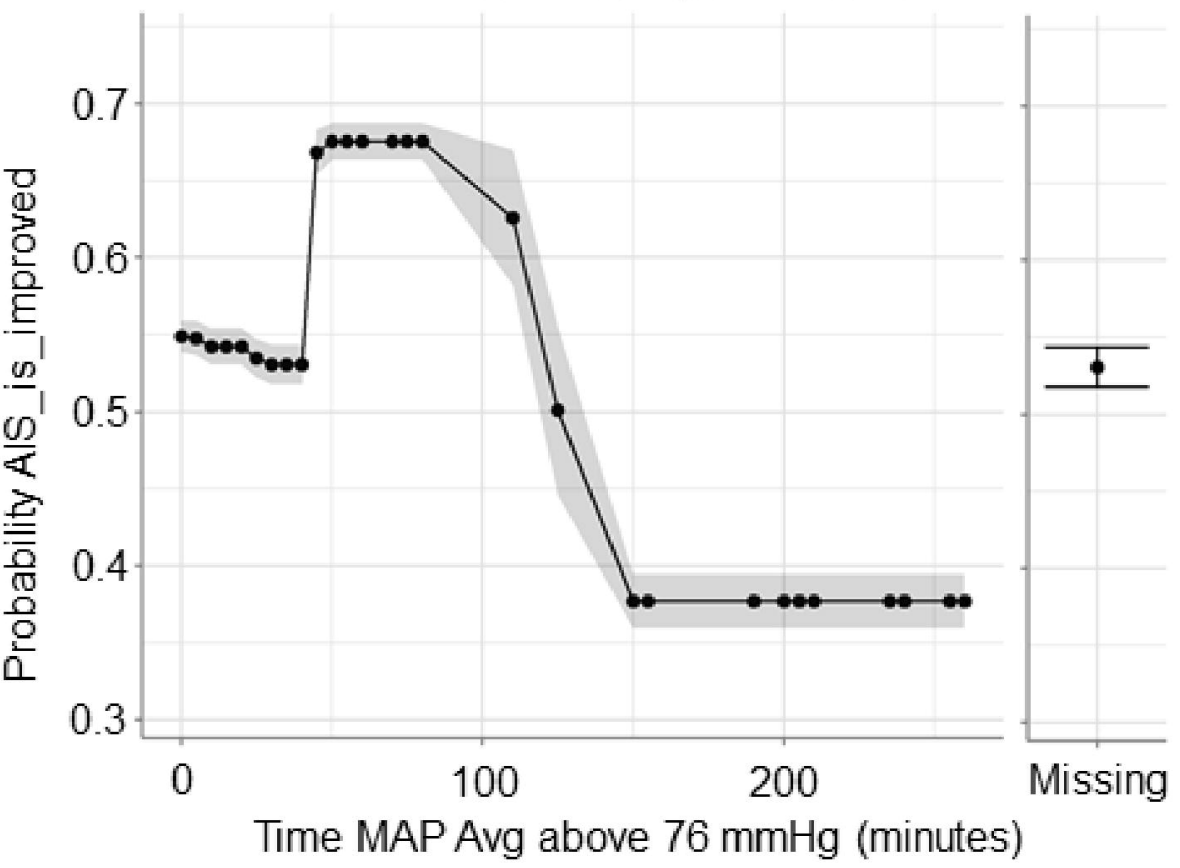

B BP XGB: $_{\text {: }}$ AlS_ad

0.7

nse to display the preprint in perpetuity. It is ma

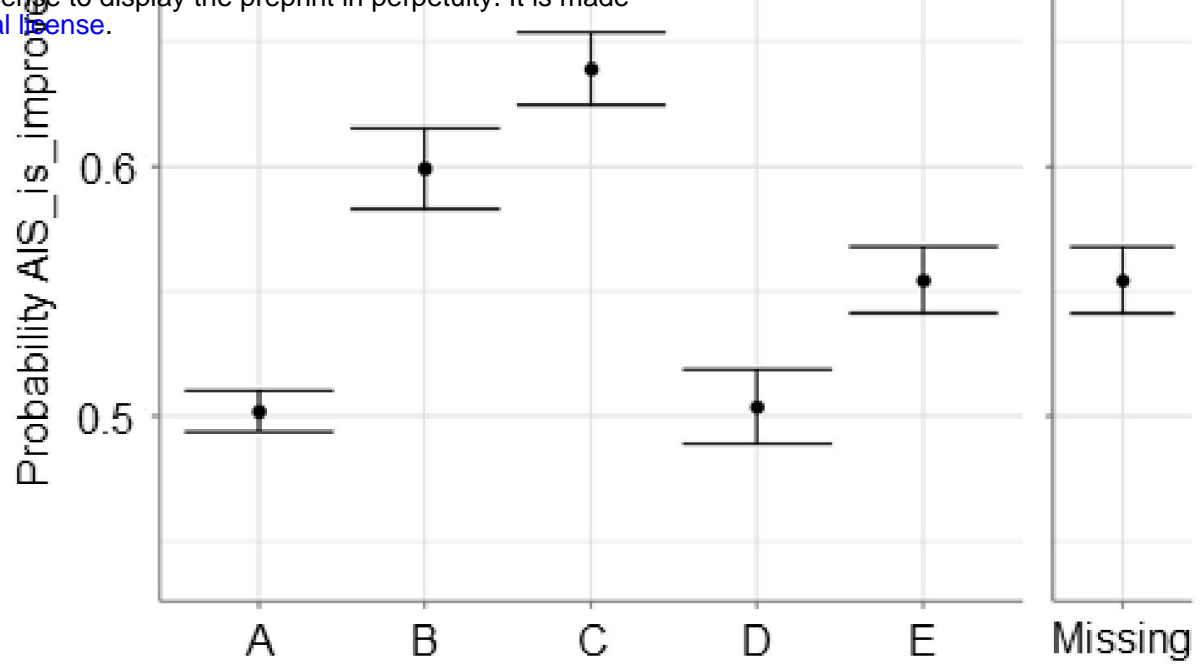

AlS at admission

D BP XGB: $_{\text {- time_MAP_above_104 }}$

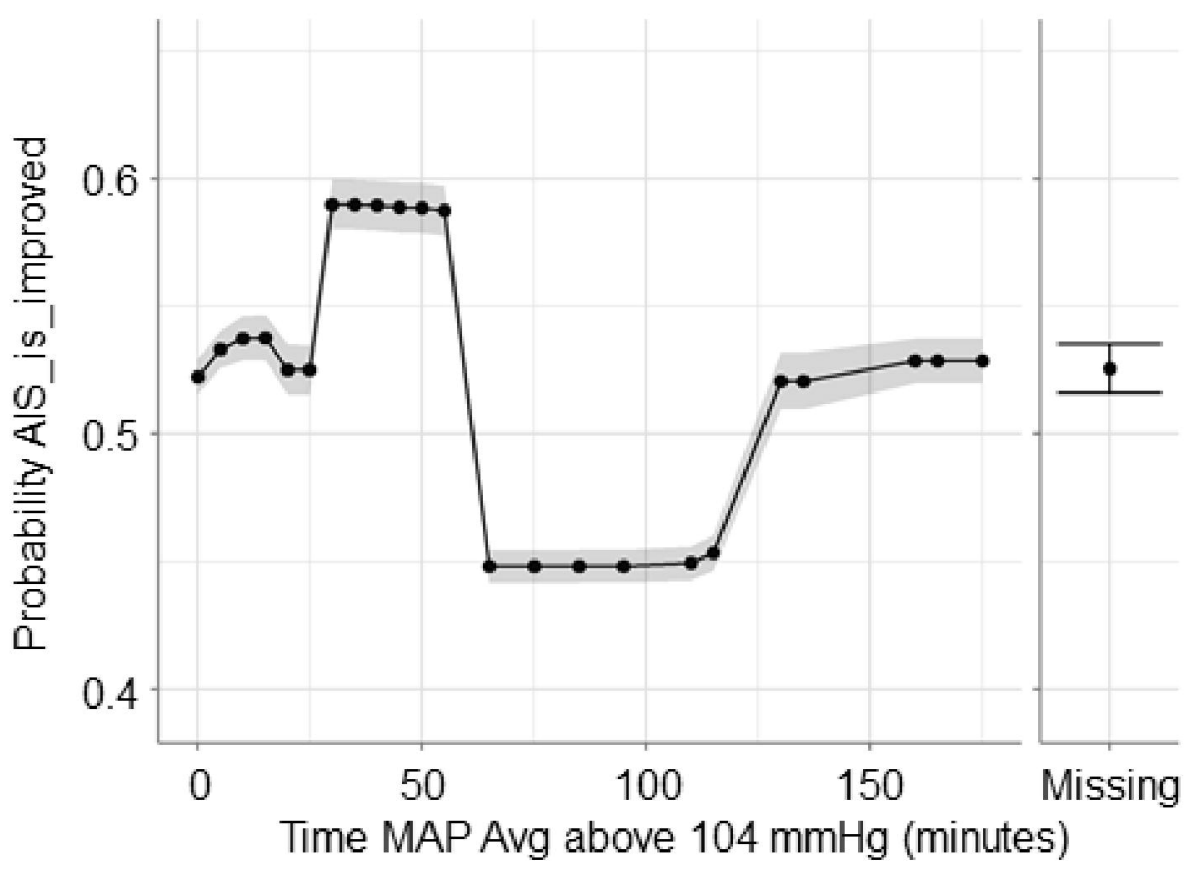

F BP XGE: $_{\text {: }}$ time_MAP_below_76

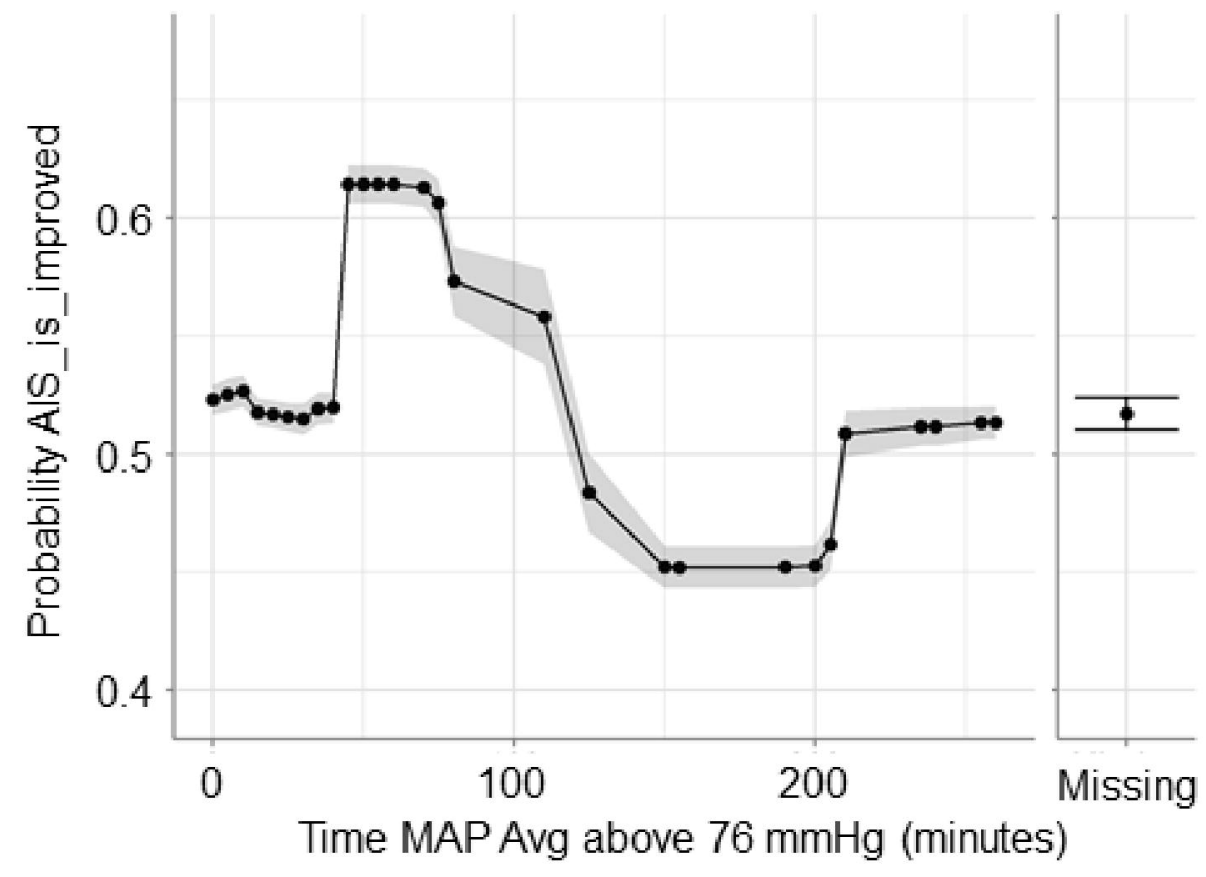




\section{Figure 7}

A $\mathrm{BP}_{\log }$ Validation

Predicted

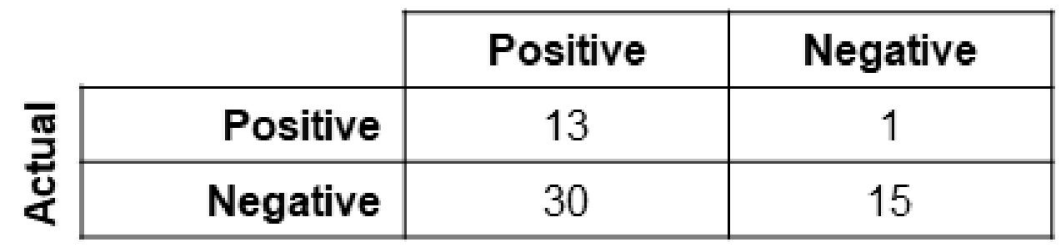

B $\mathrm{BP}_{\mathrm{XGB}}$ Validation

\begin{tabular}{|c|c|c|c|}
\hline & \multicolumn{2}{|c|}{ Predicted } \\
\hline & & Positive & Negative \\
\hline \multirow{2}{*}{$\begin{array}{l}\overline{\text { J }} \\
\text { 总 } \\
\end{array}$} & Positive & 9 & 5 \\
\hline & Negative & 31 & 14 \\
\hline
\end{tabular}

C

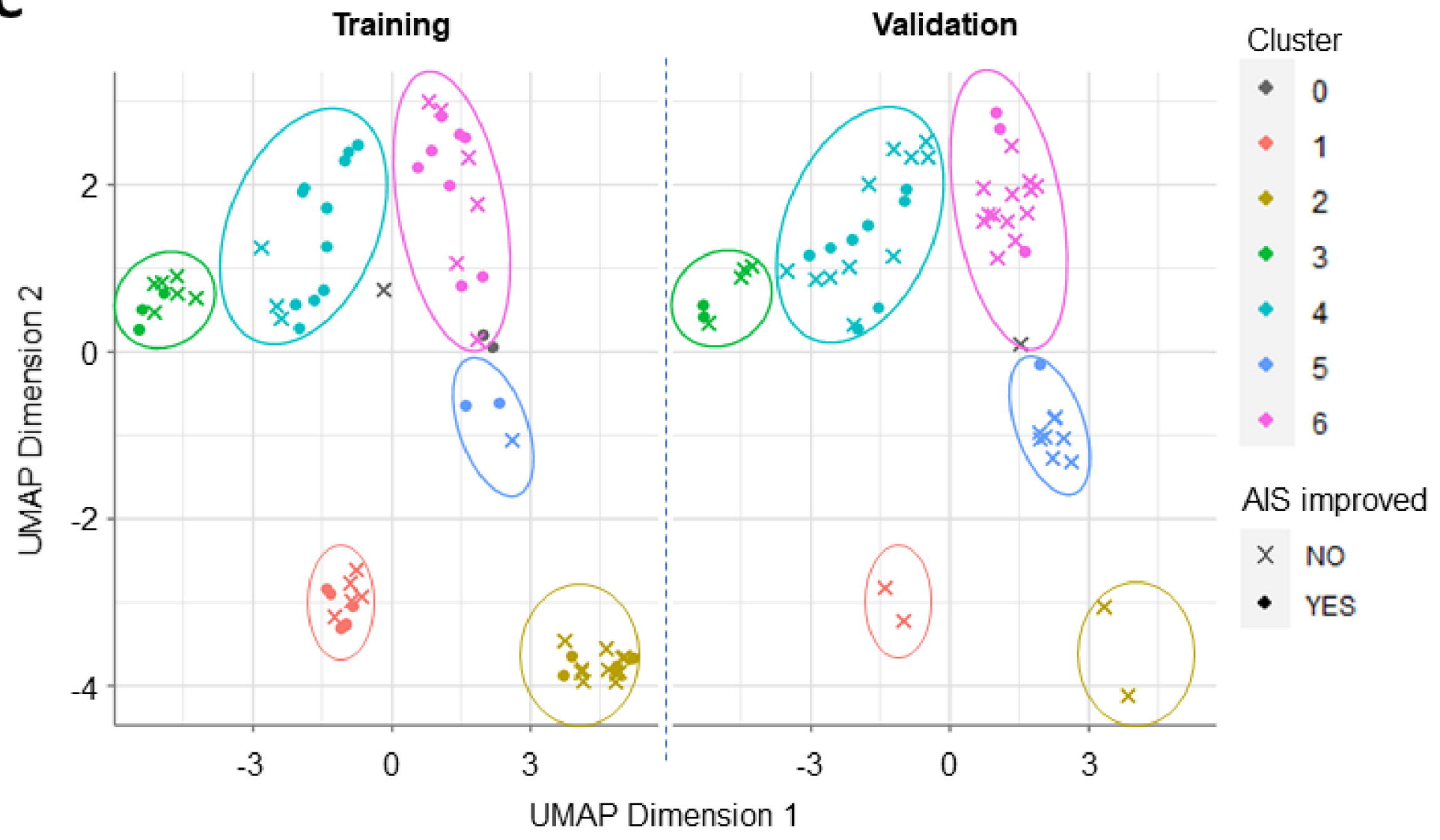


Supplementary Table 1: Features and definitions for the features used for modeling.

\begin{tabular}{|c|c|}
\hline Features & Full Name \\
\hline age & Age of patient \\
\hline Als ad & AlS score at hospital admission \\
\hline AlS dis $^{*}$ & AlS score at hospital discharge \\
\hline Anesthesia_choice_1_Sevoflurane & Whether Sevoflurane was administered \\
\hline Anesthesia_choice_2_Propofol & Whether Propofol was administered \\
\hline Anesthesia_choice_3_Fentanyl & Whether Fentanyl was administered \\
\hline Anesthesia_choice_4_Remifentanil & Whether Remifentanil was administered \\
\hline Anesthesia_choice_5_Desflurane & Whether Desflurane was administered \\
\hline Anesthesia_choice_6_Ketamine & Whether Ketamine was administered \\
\hline Central_Cord_Injury & Whether patient had a central cord injury \\
\hline Cervical_Injury & Whether patient had a cervical injury \\
\hline DiaBP_kurtosis & Intraoperative diastolic blood pressure kurtosis \\
\hline DiaBP- mean & Intraoperative diastolic blood pressure mean \\
\hline DiaBP sd & Intraoperative diastolic blood pressure standard deviation \\
\hline DiaBP skew & Intraoperative diastolic blood pressure skew \\
\hline Fracture & Whether patient had a fracture \\
\hline Hemorrhagic Injury & Whether patient had a hermorrhagic injury \\
\hline HR kurtosis & Intraoperative heart rate kurtosis \\
\hline HR_mean & Intraoperative heart rate mean \\
\hline HR_sd & Intraoperative heart rate standard deviation \\
\hline HR_skew & Intraoperative skew \\
\hline length_surg & Length of spinal cord injury surgery \\
\hline MAP_kurtosis & Intraoperative mean arterial pressure kurtosis \\
\hline MAP_mean & Intraoperative mean arterial pressure mean \\
\hline MAP_sd & Intraoperative mean arterial pressure standard deviation \\
\hline MAP_skew & Intraoperative mean arterial pressure skew \\
\hline MRI_1_BASIC_Score & MRI BASIC neuroimaging score \\
\hline OR_Dōpamine_a & Whether Dopamine was administered \\
\hline OR_Ephedrine_a & Whether Ephedrine was administered \\
\hline OR_FiO2 & Intraoperative fraction of inspired oxygen mean \\
\hline OR_Norepinephrine a & Whether Norepinephrine was administered \\
\hline OR_Phenylephrine_à & Whether Phenylephrine was administered \\
\hline OR_Polytrauma_a & Whether patient had a polytrauma \\
\hline OR_SpnalSurgryType_a & Type of spinal cord injury type \\
\hline Past_History_of_SCl_a & Whether patient had a previous spinal cord injury \\
\hline Past_History_of_TBI_a & Whether patient had a previous traumatic brain injury \\
\hline Penetrating_Injury & Whether patient had a penetrating injury \\
\hline SysBP_kurtosis & Intraoperative systolic blood pressure kurtosis \\
\hline SysBP_mean & Intraoperative systolic blood pressure mean \\
\hline SysBP_sd & Intraoperative systolic blood pressure standard deviation \\
\hline SysBP_skew & Intraoperative systolic blood pressure skew \\
\hline TBI_Present & Whether patient also had a traumatic brain injury \\
\hline time_MAP_Avg_above_104 & Time patient's MAP exceeded $104 \mathrm{mmHg}$ \\
\hline time MAP Avg below 76 & Time patient's MAP fell below $76 \mathrm{mmHg}$ \\
\hline Time to OR a & Time between hospital admission and patient entered surgery \\
\hline Vertébral_Artery_Injury & Whether patient had a vertebral artery injury \\
\hline Year of Injury a & Year patient was admitted to the hospital (de-identified) \\
\hline
\end{tabular}

*excluded from the feature list for modeling 


\section{Supplementary Figure 1}

A $\mathrm{BP}_{\log }$

time_MAP_Avg_below_76 time_MAP_Avg_above_104 DiaBP_skew Time_to_OR_a MRI_1_BASIC_Score HR_skew MAP_kurtosis HR_kurtosis SysBP_sd HR_sd MAP_skew MAP mean Cervical_njury SysBP_mean HR_mean TBI_Present age length_surg DiaBP_mean DiaBP_kurtosis MAP_sd Als_ad

SysBP_kurtosis Vertebral_Artery_Injury Anesthesia_choice_3_Fentanyl SysBP_skew Anesthesia_chooice_2_Propofol

Year_of_Injury_a Past_History_of_TBI_a Past_History_of_SCl_a DiaBP_sd Fracture

Anesthesia_choice_1_Sevoflurane OR_Polytrauma_a Anésthesia_chócée_4_Remifentänil OR_Ephedrine_a OR_Norepinephrine_a OR_Phenylephrine_a Central Cord Injury OR_FiO2

Anesthesia_choice_6_Ketamine Penetrating_Injury Hemorrhagic_Injury OR_Dopamine_a OR_SpnalSurgryType_a Anesthesia_choice_5_Desflurane

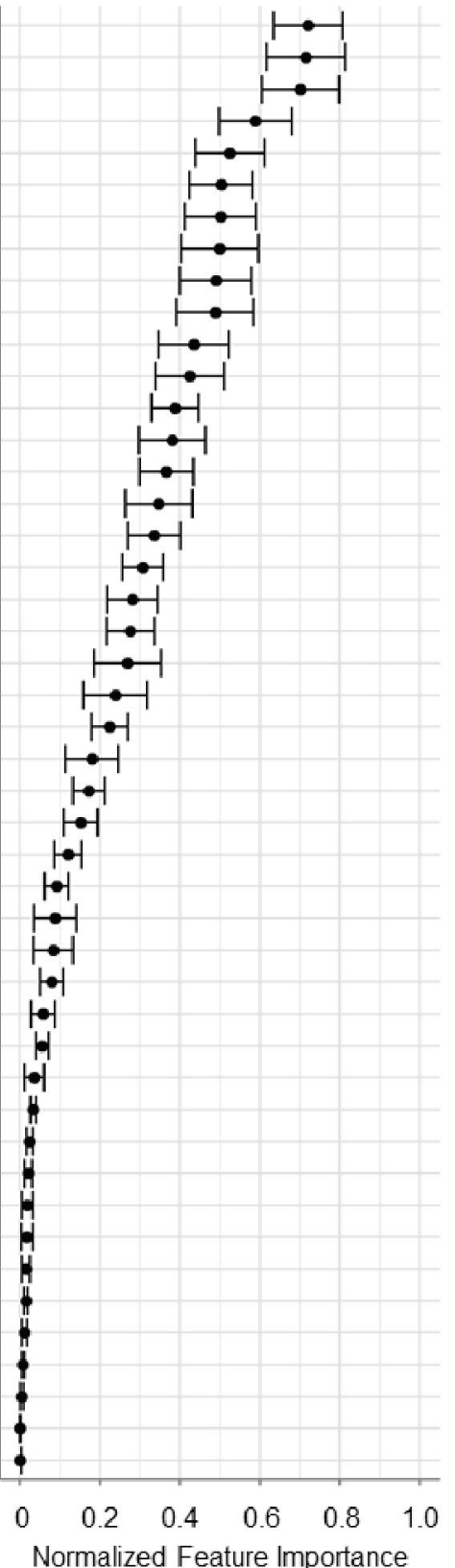

B $\mathrm{BP}_{\mathrm{XGB}}$

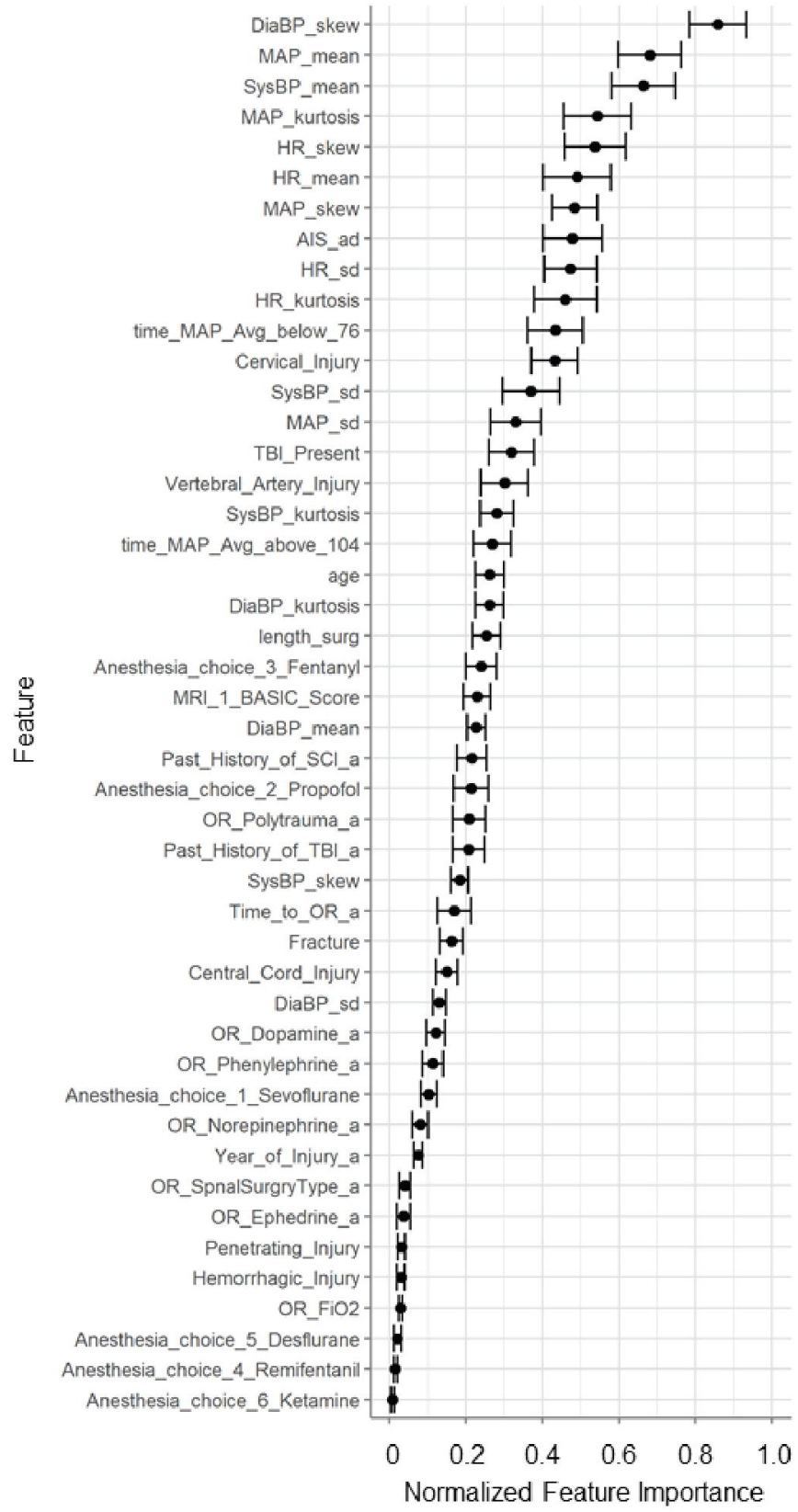




\section{Supplementary Figure $\mathbf{4}$}

A ${ }_{B P_{b o}}$

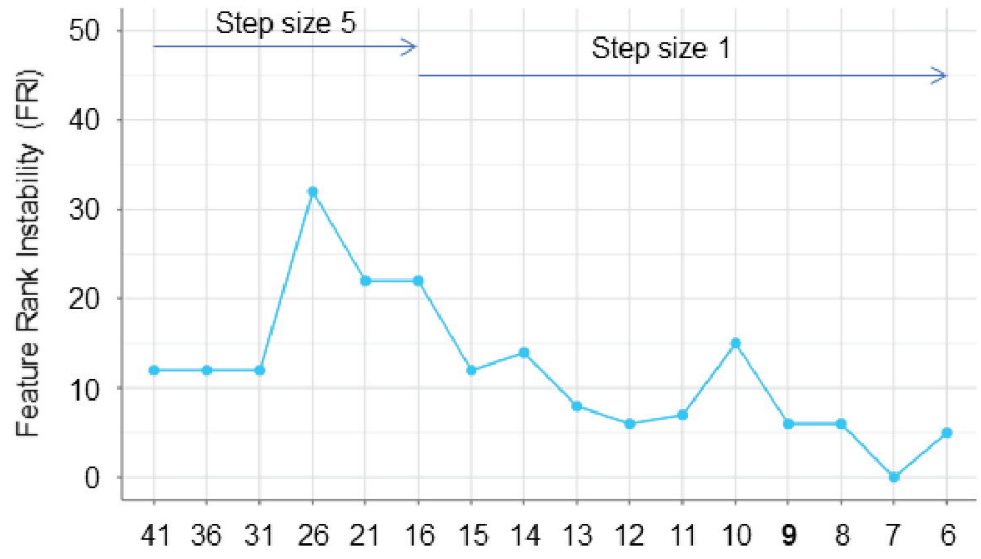

Feature List Size

B ${ }_{B P_{X B B}}$

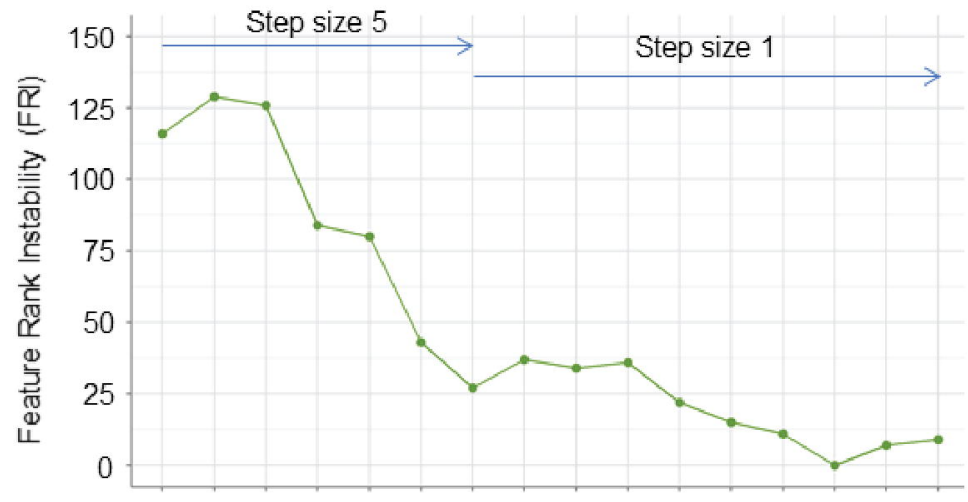

$\begin{array}{llllllllllllllll}41 & 36 & 31 & 26 & 21 & 16 & 15 & 14 & 13 & 12 & 11 & 10 & 9 & 8 & 7 & 6\end{array}$

Feature List Size 


\section{Supplementary Figure 5}

E
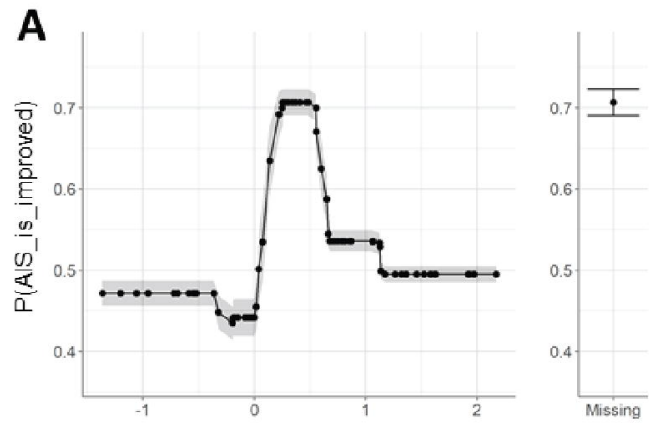

B

C

DiaBP_skew
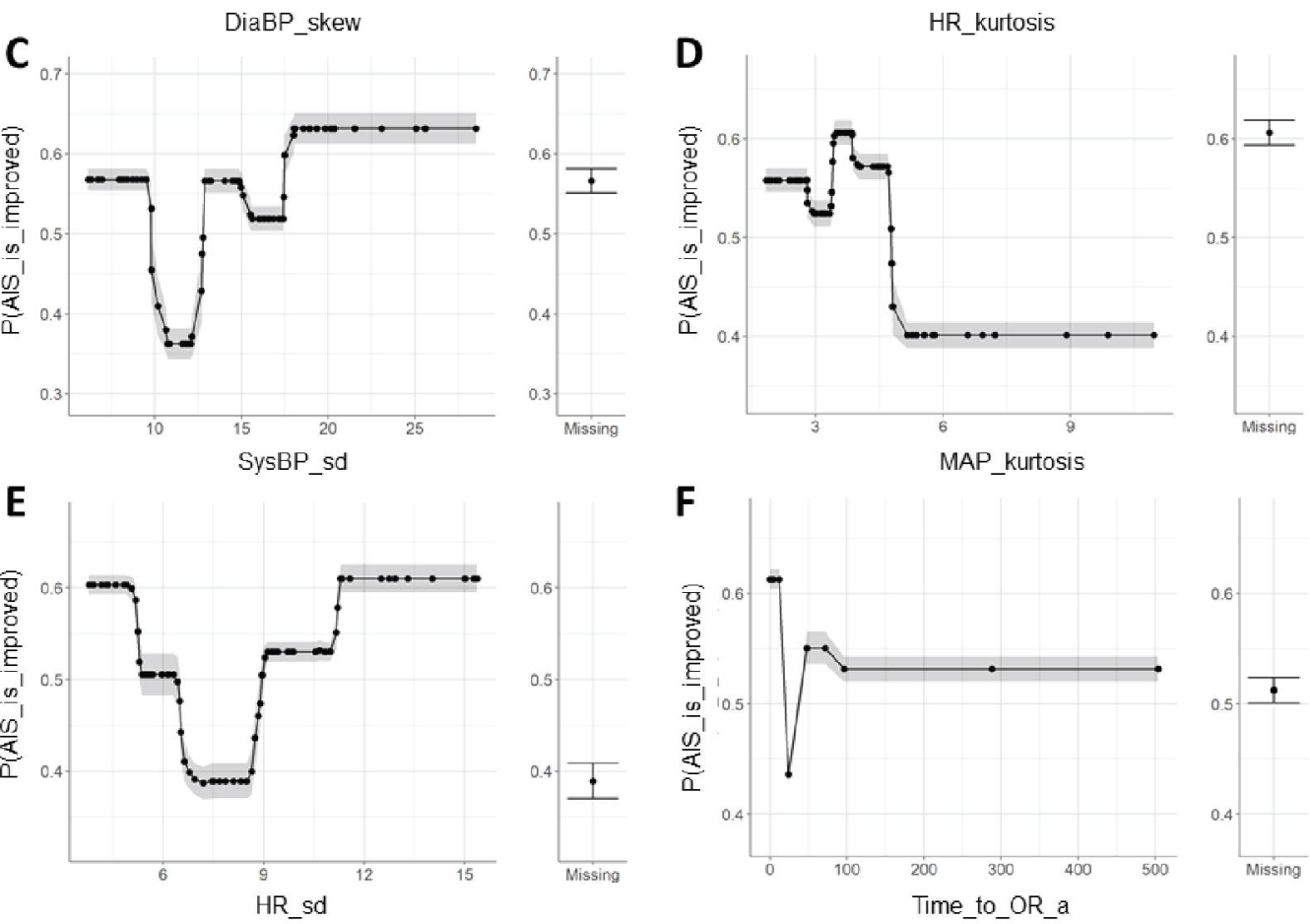

F

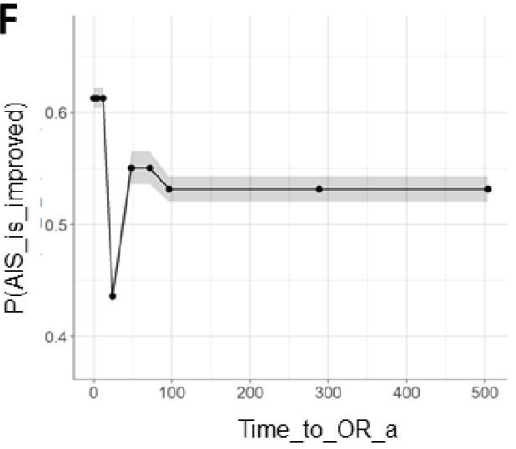

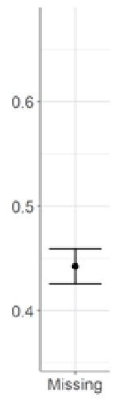

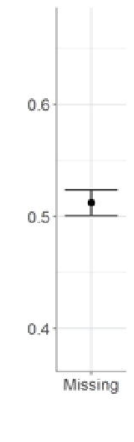

HR_sd
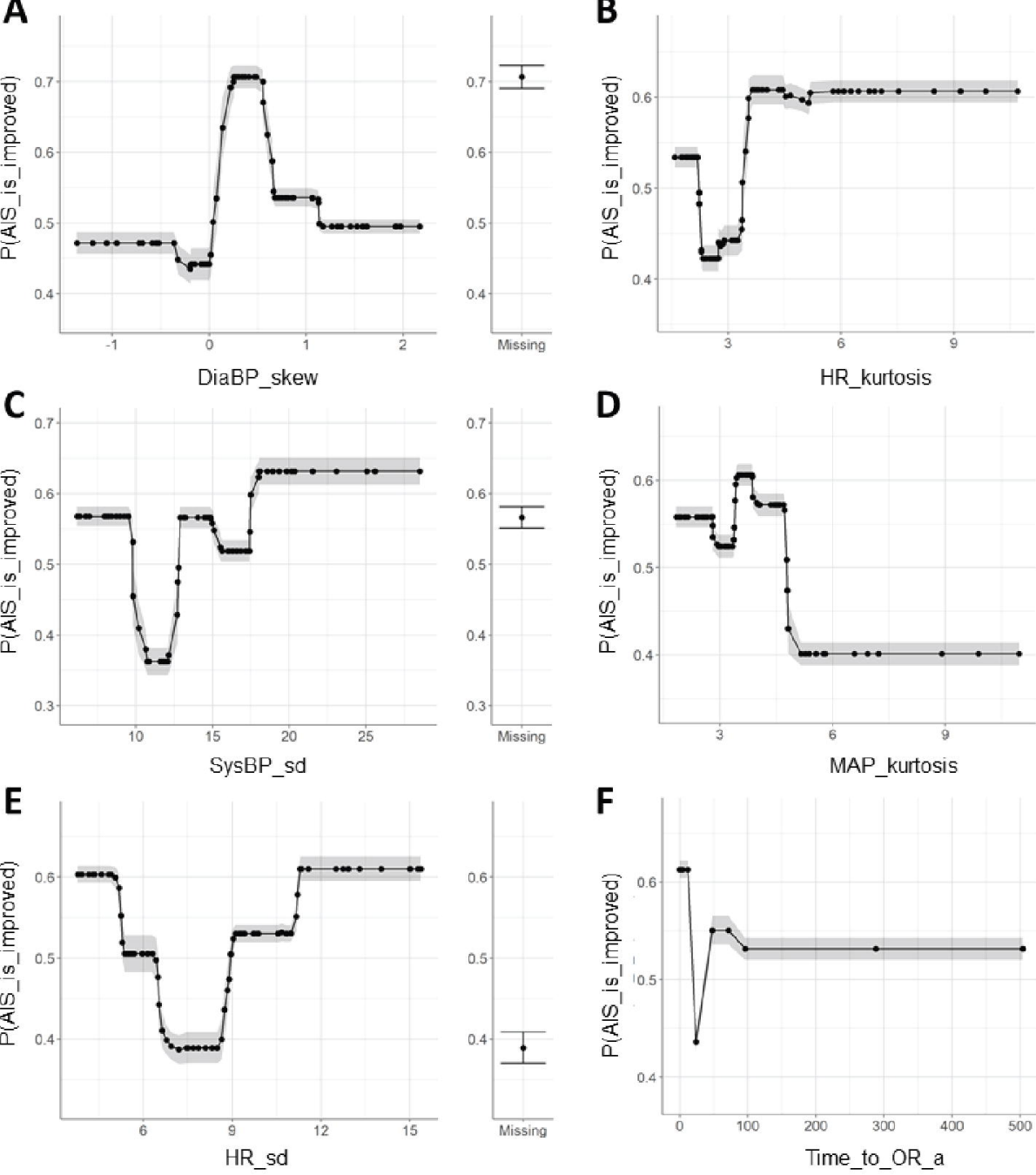


\section{Supplementary Figure 7}

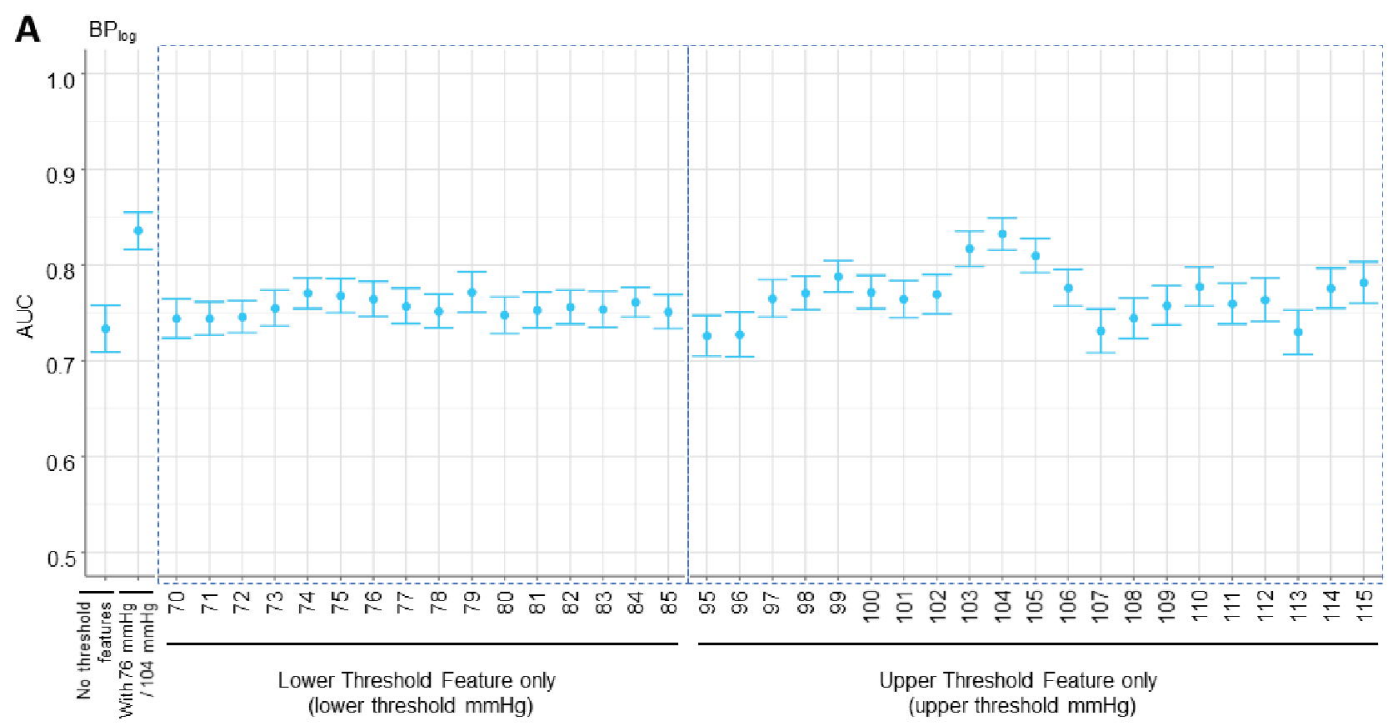

B $\quad \mathrm{PP}_{\mathrm{XGE}}$

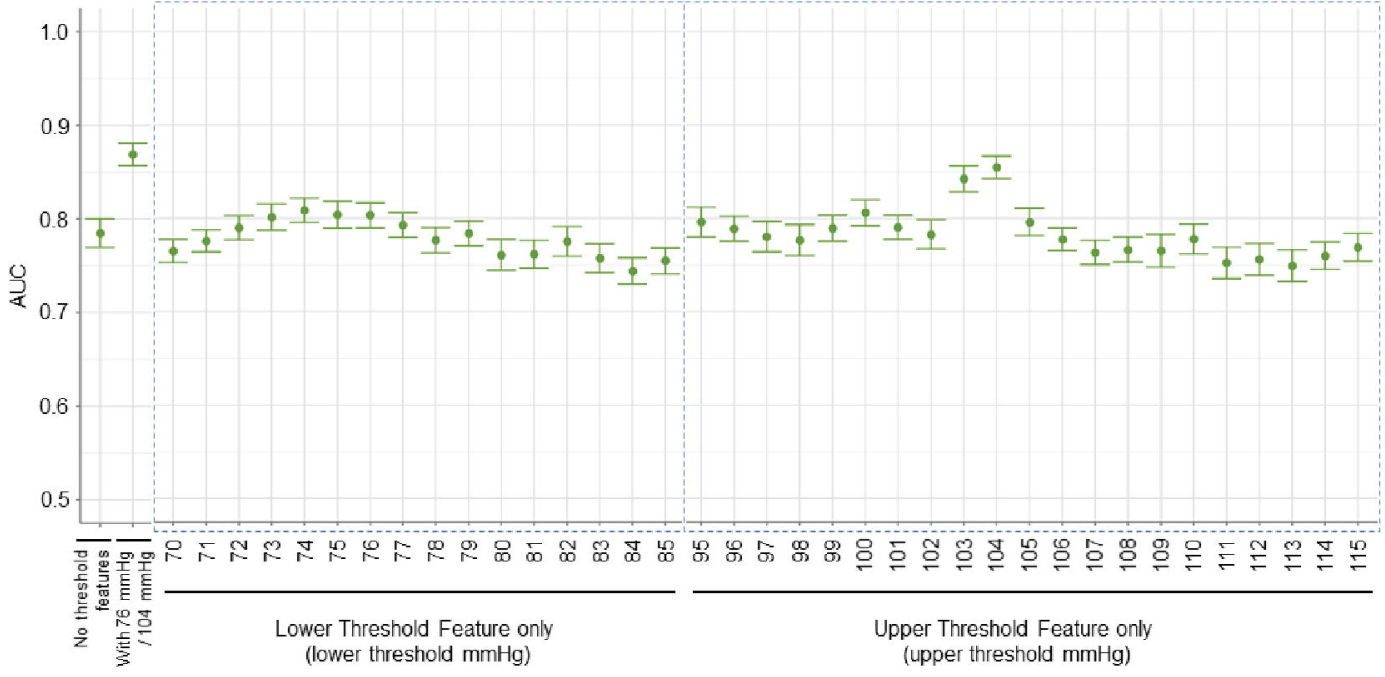




\section{Supplementary Figure 8}

A $\mathrm{BP}_{\log }$ : full feature list without MAP threshold features

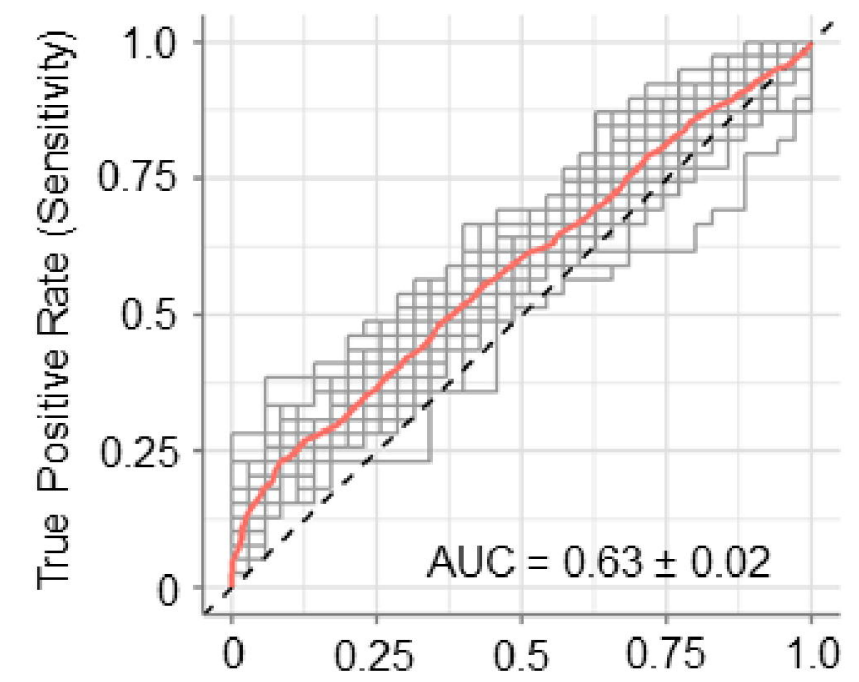

False Positive Rate (1-Specificity)

D $\quad \mathrm{BP}_{\text {log: }}$ parsimonious feature list using MAP thresholds $79 \mathrm{mmHg}$ and $104 \mathrm{mmHg}$

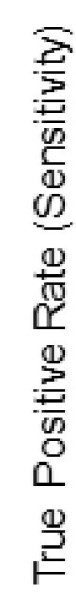

B $\mathrm{BP}_{\text {log- }}$ - full feature list with MAP threshold features

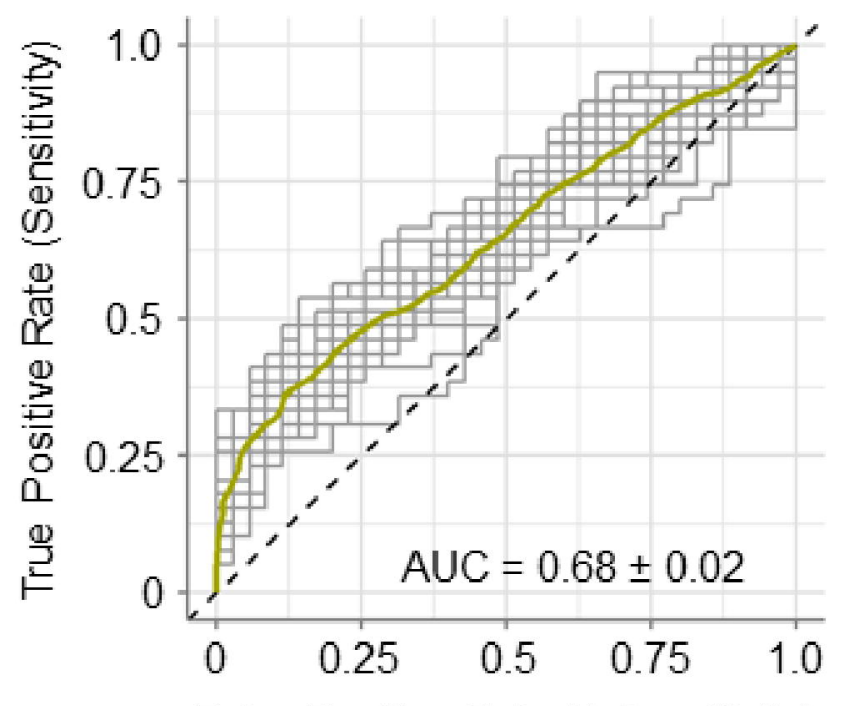

False Positive Rate (1-Specificity)
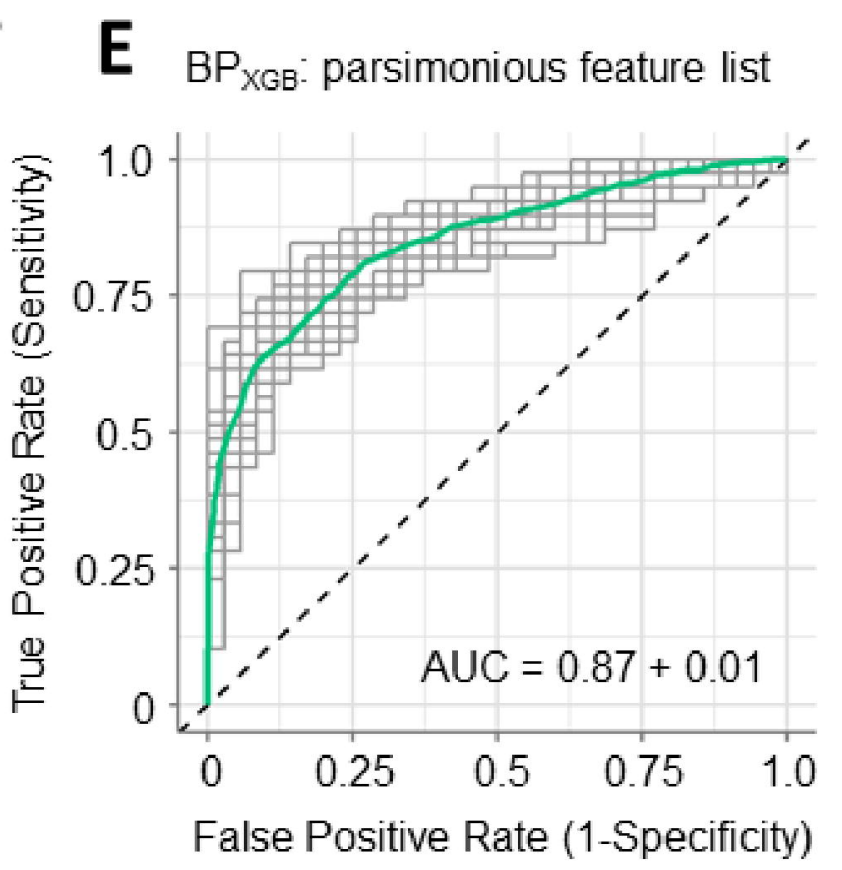

C

$\mathrm{BP}_{\text {log: }}$ : parsimonious feature list

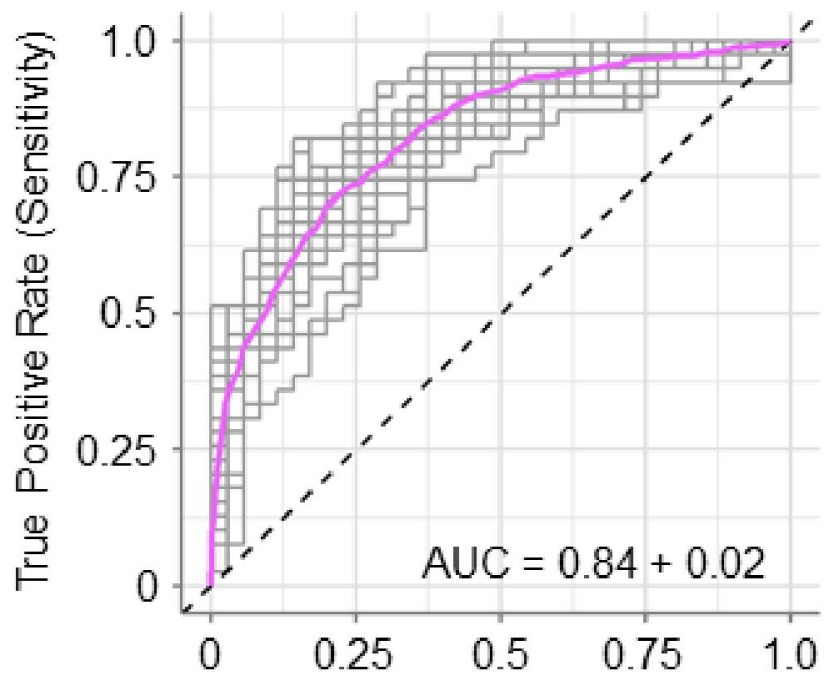

False Positive Rate (1-Specificity)

Averaqe ROCs

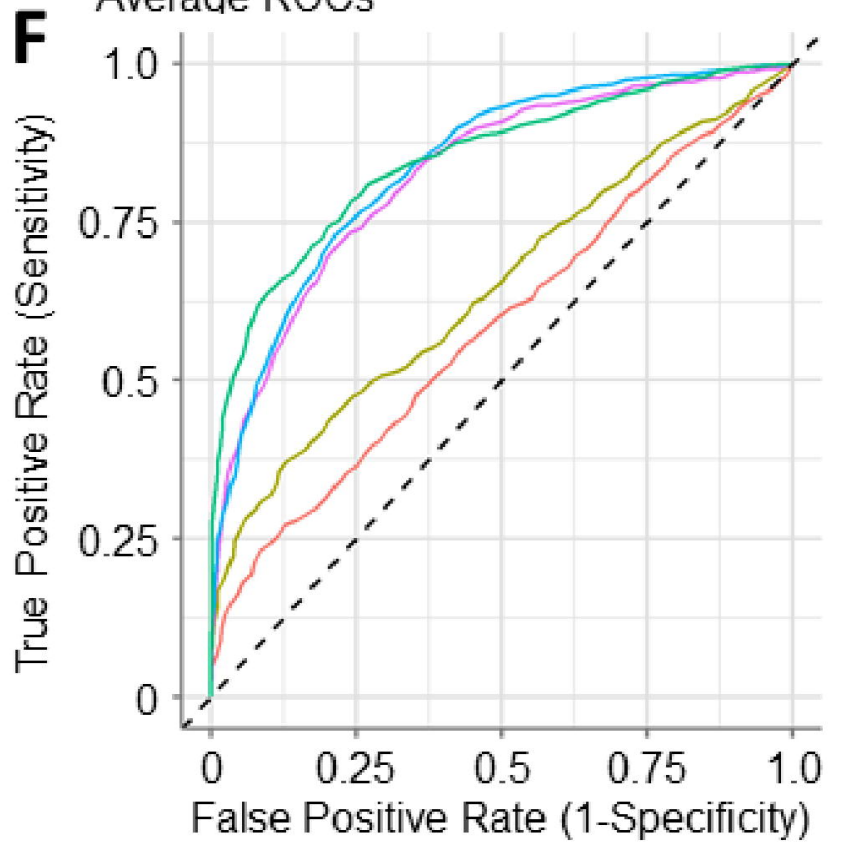

False Positive Rate (1-Specificity) 


\section{Supplementary Figure 9}

A $\mathrm{BP}_{\log }$

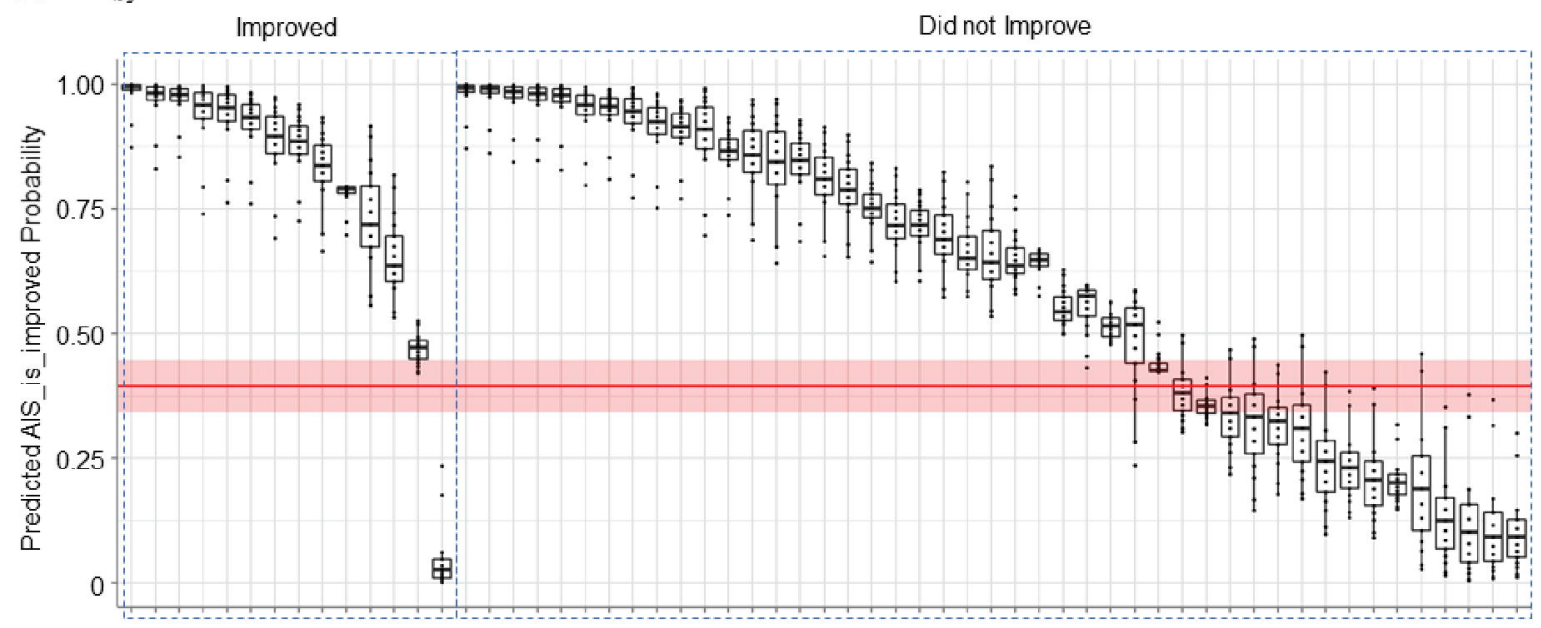

Individual Patient

B $\mathrm{BP}_{\mathrm{XGB}}$

Improved

Did not Improve

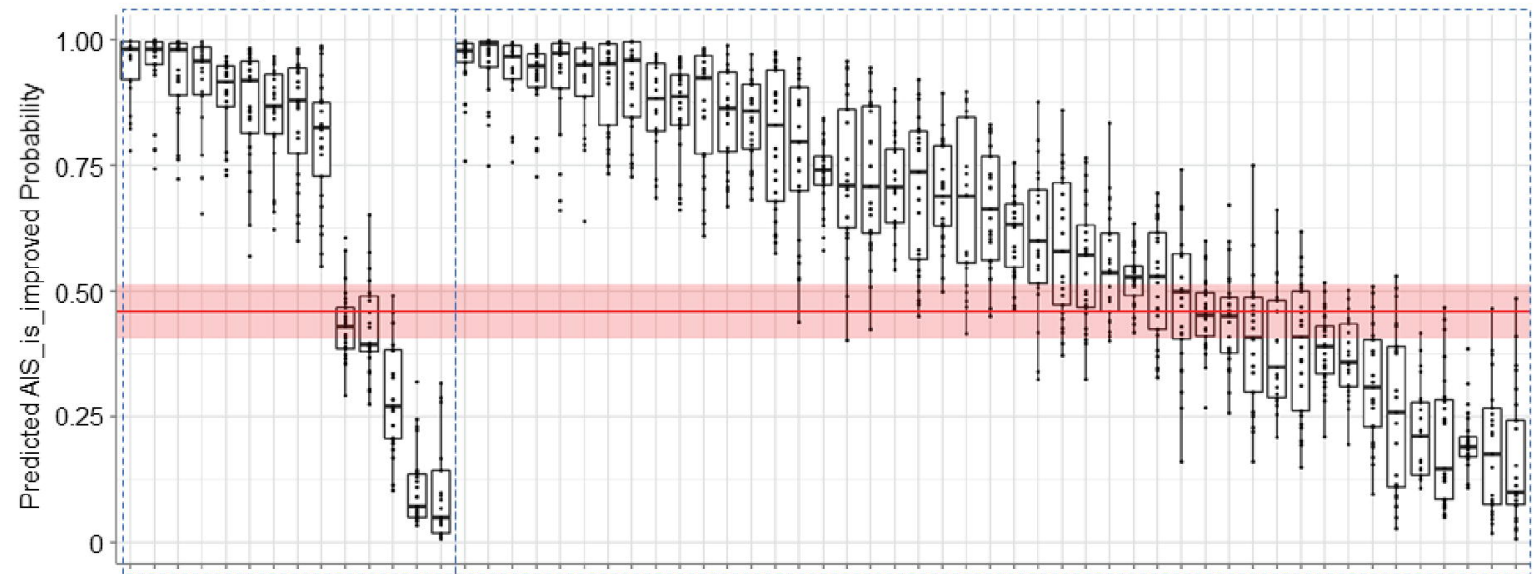


Supplementary Table 2: Population stability index (PSI) of the parsimonious $\mathrm{BP}_{\mathrm{lag}}$ and $\mathrm{BP}_{\mathrm{XGB}}$ model features.

\begin{tabular}{|c|c|c|c|}
\hline Feature & PSI & Model & Degree of Drift \\
\hline Time_to_OR_a & 0.468 & $\mathrm{BP}_{\log }$ & Significant \\
\hline MRI_1_BASIC_Score & 0.385 & $\mathrm{BP}_{\log }$ & Significant \\
\hline MAP_mean & 0.356 & $\mathrm{BP}_{\mathrm{XGB}}$ & Significant \\
\hline HR_mean & 0.354 & $\mathrm{BP}_{\mathrm{XGB}}$ & Significant \\
\hline HR_kurtosis & 0.353 & $B P_{\log }$ & Significant \\
\hline MAP_kurtosis & 0.297 & $\mathrm{BP}_{\log ,} \mathrm{BP} \times \mathrm{XGB}$ & Significant \\
\hline SysBP_sd & 0.289 & $B P_{\log }$ & Significant \\
\hline HR_sd & 0.277 & $\mathrm{BP}_{\log ,}, \mathrm{BP}_{\mathrm{XGB}}$ & Significant \\
\hline Als_ad & 0.247 & $\mathrm{BP}_{\mathrm{XGE}}$ & Maderate \\
\hline time_MAP_Avg_above_104 & 0.239 & $\mathrm{BP}_{\log }, \mathrm{BP} \times \mathrm{XGB}$ & Moderate \\
\hline time_MAP_Avg_below_76 & 0.201 & $\mathrm{BP}_{\log }, \mathrm{BP} \mathrm{XGB}_{\mathrm{X}}$ & Moderate \\
\hline DiaBP_skew & 0.175 & $\mathrm{BP}_{\log ,}, \mathrm{BP} \mathrm{XGB}_{\mathrm{X}}$ & Moderate \\
\hline Cervical Injury & 0.103 & $\mathrm{BP}_{\mathrm{XGB}}$ & Moderate \\
\hline Vertebral_Artery_Injury & 0.027 & $\mathrm{BP}_{\mathrm{XGB}}$ & Negligible \\
\hline TBI_Present & 0.002 & $\mathrm{BP}_{\mathrm{XGB}}$ & Negligible \\
\hline
\end{tabular}


Supplementary Table 3: Within-cluster mean and $95 \%$ confidence interval of numeric features.

\begin{tabular}{|c|c|c|c|c|c|c|c|c|c|c|c|c|}
\hline Cluster & $\begin{array}{c}\text { Training } \\
\mathrm{n}\end{array}$ & $\begin{array}{c}\text { Validation } \\
\mathbf{n}\end{array}$ & Time_to_OR_a & MAP_mean & HR_mean & HR_kurtosis & MAP_kurtosis & SysBP_sd & HR_sd & $\begin{array}{c}\text { time_MAP_Avg_ } \\
\text { above_104 }\end{array}$ & $\begin{array}{c}\text { time_MAP_Avg_ } \\
\text { below_76 }\end{array}$ & DiaBP_skew \\
\hline 0 & 3 & 1 & $12.95 \pm 8.15$ & $87.16 \pm 2.89$ & $86.64 \pm 5.98$ & $3.94 \pm 2.49$ & $3.67 \pm 0.76$ & $12.65 \pm 2.79$ & $10.51 \pm 3.19$ & $12.5 \pm 11.66$ & $37.5 \pm 6.33$ & $-0.6 \pm 0.38$ \\
\hline 1 & 11 & 2 & $140.15 \pm 72.32$ & $89.58 \pm 2.17$ & $73.01 \pm 5.85$ & $3.55 \pm 0.86$ & $3.27 \pm 0.55$ & $14.6 \pm 3.01$ & $8.28 \pm 1.98$ & $34.23 \pm 13.45$ & $38.46 \pm 11.82$ & $0.34 \pm 0.44$ \\
\hline 2 & 15 & 2 & $42.42 \pm 14.24$ & $76.23 \pm 2.35$ & $79.23 \pm 5.38$ & $4.17 \pm 1.21$ & $4.18 \pm 1.23$ & $14.15 \pm 2.16$ & $8.11 \pm 1.33$ & $4.12 \pm 3.78$ & $194.41 \pm 43.64$ & $0.62 \pm 0.42$ \\
\hline 3 & 9 & 6 & $27.49 \pm 15.13$ & $99.65 \pm 2.74$ & $72.85 \pm 4.81$ & $4.15 \pm 1.72$ & $3.54 \pm 0.48$ & $18.54 \pm 2.86$ & $8.59 \pm 2.33$ & $136.67 \pm 17.57$ & $17.67 \pm 13.14$ & $0.57 \pm 0.34$ \\
\hline 4 & 14 & 19 & $13.06 \pm 2.49$ & $92.92 \pm 1.16$ & $73.53 \pm 3.44$ & $4.54 \pm 1.01$ & $5.13 \pm 1.11$ & $15.21 \pm 1.4$ & $7.82 \pm 1.05$ & $46.97 \pm 5.46$ & $17.27 \pm 6.57$ & $0.41 \pm 0.21$ \\
\hline 5 & 3 & 9 & $11.85 \pm 5.12$ & $82.66 \pm 3.51$ & $78.55 \pm 9.31$ & $3.81 \pm 1.46$ & $2.98 \pm 0.46$ & $17.93 \pm 4.81$ & $8.42 \pm 2.12$ & $11.67 \pm 5.17$ & $62.92 \pm 6.32$ & $0.22 \pm 0.31$ \\
\hline 6 & 14 & 16 & $15.4 \pm 3.24$ & $88.92 \pm 1.16$ & $73.9 \pm 3.94$ & $4.65 \pm 1.06$ & $4.93 \pm 0.98$ & $12.64 \pm 1.6$ & $7.45 \pm 1.2$ & $8 \pm 2.23$ & $14.17 \pm 3.93$ & $0.12 \pm 0.37$ \\
\hline
\end{tabular}

